\title{
Correlating functional near-infrared spectroscopy with underlying cortical regions of 0-, 1-, and 2-year-olds using theoretical light propagation analysis
}

\author{
Lin Cai $\odot,{ }^{\text {a }}$ Eiji Okada $\odot,{ }^{\text {a }}$ Yasuyo Minagawa, ${ }^{\text {b }}$ and Hiroshi Kawaguchi $\odot^{\text {a,c, } *}$ \\ ${ }^{a}$ Keio University, Department of Electronics and Electrical Engineering, Yokohama, Japan \\ ${ }^{b}$ Keio University, Department of Psychology, Tokyo, Japan \\ ${ }^{c}$ National Institute of Advanced Industrial Science and Technology, \\ Human Informatics and Interaction Research Institute, Tsukuba, Japan
}

\begin{abstract}
Significance: The establishment of a light propagation analysis-based scalp-cortex correlation (SCC) between the scalp location of the source-detector (SD) pair and brain regions is essential for measuring functional brain development in the first 2 years of life using functional nearinfrared spectroscopy (fNIRS).

Aim: We aimed to reveal the optics-based SCC of 0-, 1-, and 2-year-olds (yo) and the suitable SD distance for this age period.

Approach: Light propagation analyses using age-appropriate head models were conducted on SD pairs at 10-10 fiducial points on the scalp to obtain optics-based SCC and its metrics: the number of corresponding brain regions $\left(N_{C B R}\right)$, selectivity and sensitivity of the most likely corresponding brain region (MLCBR), and consistency of the MLCBR across developmental ages. Moreover, we assessed the suitable SD distances for 0-, 1-, and 2-yo by simultaneously considering the selectivity and sensitivity of the MLCBR.
\end{abstract}

Results: Age-related changes in the SCC metrics were observed. For instance, the $N_{C B R}$ of 0 -yo was larger than that of 1- and 2-yo. Conversely, the selectivity of 0-yo was lower than that of 1- and 2-yo. The sensitivity of 1-yo was higher than that of 0-yo at 15- to 30-mm SD distances and higher than that of 2-yo at 10-mm SD distance. Notably, the MLCBR of the fiducial points around the longitudinal fissure was inconsistent across age groups. An SD distance between 15 and $25 \mathrm{~mm}$ was found to be appropriate for satisfying both sensitivity and selectivity requirements. In addition, this work provides reference tables of optics-based SCC for 0-, 1-, and 2-yo.

Conclusions: Optics-based SCC will be informative in designing and explaining child developmental studies using fNIRS. The suitable SD distances were between 15 and $25 \mathrm{~mm}$ for the first 2 years of life.

(C) The Authors. Published by SPIE under a Creative Commons Attribution 4.0 Unported License. Distribution or reproduction of this work in whole or in part requires full attribution of the original publication, including its DOI. [DOI: 10.1117/1.NPh.8.2.025009]

Keywords: functional near-infrared spectroscopy; light propagation; scalp-cortex correlation; early development.

Paper 21009R received Feb. 20, 2021; accepted for publication May 18, 2021; published online May 31, 2021.

\section{Introduction}

The first two years of human life are characterized by the most dynamic growth in brain structures $^{1-5}$ and remarkable cognitive and behavioral changes. ${ }^{6,7}$ Functional near-infrared spectroscopy (fNIRS; a list of abbreviations is provided in Table S1 in the Supplementary Material for the convenience of the reader) is an irreplaceable neuroimaging tool for studying early brain functional development, providing unprecedented opportunities for recording the hemodynamic

*Address all correspondence to Hiroshi Kawaguchi, hiroshi.kawaguchi@aist.go.jp 
Cai et al.: Correlating functional near-infrared spectroscopy with underlying cortical regions...

response in awake, behaving infants because of its balanced temporal-spatial resolution and resilience to movement. ${ }^{8-11}$ Notably, although the Centers for Disease Control and Prevention defines 0- to 1-year-olds (yo) and 2- to 3-yo children as infants and toddlers, respectively, we have described 0 - to 2-yo children as infants in this study for readability.

Despite the suitability of fNIRS in infant studies, a major limitation of this technique is the inability of fNIRS data to provide structural information of the head tissue. In fNIRS measurements, a source-detector (SD) pair positioned on the scalp surface measures local concentration changes in oxygenated and deoxygenated hemoglobin caused by neural activity. ${ }^{12-15}$ On the other hand, while neural activity tied to a specific human function originates in local brain regions. The absence of structural information in the fNIRS signal makes it impossible to correlate the signal response with the anatomical brain regions. Therefore, in fNIRS studies, the scalp location where the SD pair is attached to its underlying brain region where the fNIRS signal originates should be mapped. We call this mapping the scalp-cortex correlation (SCC).

Several methods for obtaining SCC have been proposed for studies on adults; however, only a few studies have provided SCC for the infant population. Similar to adults, infant SCC is mostly based on a simple geometrical technique, i.e., correlating the location of the SD pair on the scalp, typically, the midpoint of the SD pair, with cortical regions in a simple pointto-point geometrical manner. For instance, researchers often referred to the international $10-20$ or $10-10$ system ${ }^{16}$ when attaching SD pairs on the scalp and then inferred the anatomical locations ${ }^{17-19}$ according to the geometrical SCC of the adult head ${ }^{20,21}$ or infant head. ${ }^{22-25}$ Notably, by linearly reducing the size of the adult heads, the virtual registration method ${ }^{26}$ has also been employed to estimate SCC in infant studies. ${ }^{27-29}$

As described above, the point-to-point geometrical SCC provides a tolerable estimation of the underlying brain regions for the absorption change acquired by the SD pair. Nevertheless, the geometrical SCC is based on the assumption that the absorption change occurs at the cortical projection point below the midpoint between the SD pair, and light scattering in the head tissue is not considered. Mounting evidence from light propagation analysis in the adult head revealed light scattering in the head tissue could have a considerable influence on the partial pathlength (PPL) in the brain and the spatial sensitivity profile (SSP). ${ }^{30-34}$ Notably, a few studies have already demonstrated that light propagation in the infant heads is distinct from that in adults owing to structural differences. ${ }^{35,36}$ Furthermore, very recent studies on adults have started considering light propagation in turbid media when calculating the SCC. ${ }^{37,38}$ However, to date, no light propagation analysis-based SCC data are available for 0- to 2-yo infants. In addition to age, SD distance must have a significant influence on optics-based SCC during early development. Only a few studies have examined the effect of SD distance on fNIRS sensitivity in infant brain tissue. ${ }^{35,39}$ For example, Fukui et al. ${ }^{35}$ found that fNIRS sensitivity to gray matter (GM) and white matter (WM) of neonates was modulated by the SD distance. These threads of evidence revealed that it remains largely unknown how age and SD distance affect optics-based SCC in 0- to 2-yo infants and how to choose an appropriate SD distance to ensure both the sensitivity to cerebral hemodynamics and the selectivity of signals from a specific brain region of interest. To address these issues, the current study aimed to create a precise optics-based SCC between SD pairs on the scalp fiducial point and brain regions defined by a macro-anatomical atlas by considering the light scattering in $0-, 1-$, and 2-yo infant heads. In addition, we quantitatively characterized the changes in SCC with age and SD distance and evaluated the suitable SD distances for each age. The optics-based SCC was obtained for each SD pair by solving the diffusion equation.

\section{Materials and Methods}

\subsection{Infant Head Structure and AAL Atlas}

The anatomical head structure of the infants and the corresponding brain atlas used in this study were obtained from publicly available data. ${ }^{40}$ In the present study, we used age-appropriate average structural images acquired with a $3 \mathrm{~T}$ magnetic resonance imaging (MRI) scanner. A set of 
longitudinal images of 95 healthy infants [56 males and 39 females, gestational age at birth: $37.9 \pm 1.8$ (mean \pm standard deviation) weeks] were scanned three times when their postmenstrual age was $41.5 \pm 1.7,94.2 \pm 3.4$, and $146.2 \pm 4.9$ weeks, respectively. Based on the difference in subtracting gestational age from postmenstrual age, the population used in this study could be divided into three age groups concentrating around 0,1 , and 2 years of age. All participants in our dataset had normal fetal ultrasound during pregnancy and were free of congenital anomalies, metabolic disease, and focal lesions after birth. T2-weighted images were obtained with a voxel size of $1.25 \times 1.25 \times 1.95 \mathrm{~mm}^{3}$ for 0 -yo and T1-weighted images were obtained with a voxel size of $1 \times 1 \times 1 \mathrm{~mm}^{3}$ for 1- and 2-yo. The tissue probability maps of GM, WM, and cerebrospinal fluid (CSF) that exhibited similar geometry to the structural images were also used for head tissue segmentation (see Sec. 2.2.1).

To obtain the optics-based SCC between the fiducial points and brain regions, we chose the automated anatomical labeling (AAL) atlas ${ }^{41}$ to parcellate the infant's brain. The AAL atlas is widely used in cognitive neuroscience. In addition, remarkably, it parcellates a human brain into multiple non-overlapping regions according to the identification of main sulci, which are already clearly visible from birth and preserved throughout normal brain development. ${ }^{22}$ We used the AAL atlas with 90 brain regions (Table S2 in the Supplementary Material) in the infant space, which maintains the consistency of the AAL map propagation from the adult Colin 27 brain to the infant images using indirect fusion approach and a feature-based groupwise registration algorithm (see Ref. 40). Infant atlases from 0-, 1-, and 2-yo were built using infant MRI segmentation and groupwise registration methods. The atlases are publicly available on the NITRC website. ${ }^{42}$

\subsection{Light Propagation Analysis}

\subsubsection{Construction of age-appropriate structural head models}

We can use an age-appropriate template of the head to substitute the subject-specific head anatomy to localize the macroanatomical structure when individual infant MRIs are not available. ${ }^{24,25}$ Hence, we constructed three age-appropriate head models by segmenting the tissues of average MRI images from 0-, 1-, and 2-yo infants for further light propagation analysis. Specifically, a semiautomatic approach was used to segment the MRI images into air and five types of head tissues with different optical properties. First, the head masks, i.e., the air/scalp boundaries, were extracted from the average T2-weighted images for 0-yo, and T1-weighted images for 1- and 2-yo using a simple thresholding method. Second, the tissue probability maps of the CSF, GM, and WM were converted to binary intracranial regions in each image. It was difficult to identify the scalp/skull and skull/CSF boundaries in the average MRI images because of the thin structures of the scalp and skull and blurring caused by the averaging of slightly misaligned multi-subject images. Thus, we applied morphological operations to extract the scalp/skull and skull/CSF boundaries based on the representative thickness of the scalp (3.5, 4, and $4 \mathrm{~mm}$ for 0-, 1-, and 2-yo, respectively) and skull $(2.2,3$, and $3.8 \mathrm{~mm}$ for 0 -, $1-$, and 2-yo, respectively), as described in the literature. ${ }^{43,44}$ Finally, five-layered head models of 0-, 1-, and 2-yo infants were created by integrating the above intracranial regions and boundaries of superficial tissues. Contradictions in the integration process were manually and/or automatically corrected. The age-appropriate five-layered models for the three age groups are shown in Fig. 1.

\subsubsection{Light propagation calculation and optical property}

The volumetric tetrahedral mesh for each age-appropriate head model was generated using the iso 2 mesh toolbox ${ }^{45}$ for light propagation analysis by employing the diffusion equation along with the finite element method. Similar to a previous study, ${ }^{46}$ we confirmed that the quality of the created volumetric tetrahedral mesh was sufficient for conducting the light propagation analysis. Specifically, we calculated the number of nodes, elements, and faces in each mesh model and further computed the Joe-Lie quality index, ${ }^{47} q_{v o l}$, for every tetrahedron for all three ages using the following equation: 
Cai et al.: Correlating functional near-infrared spectroscopy with underlying cortical regions...
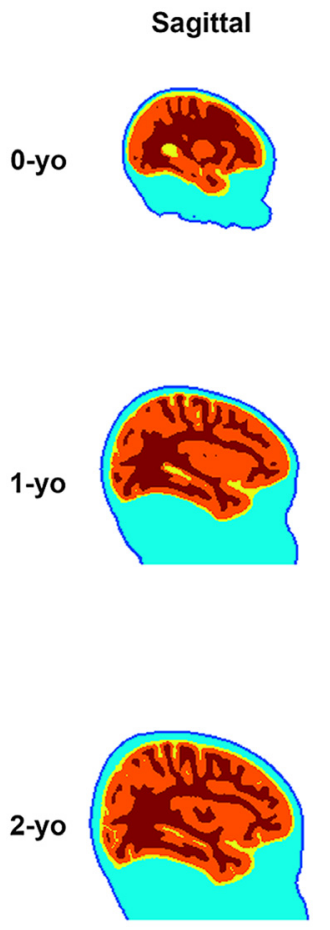

Scalp
Skull

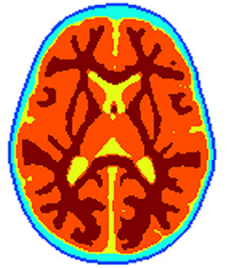

(b)

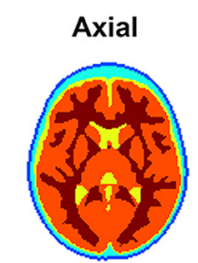

(a)

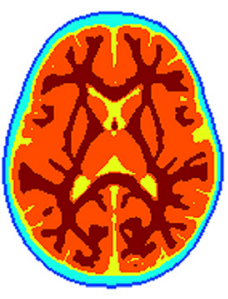

CSF $\square$ Gray matter
Coronal
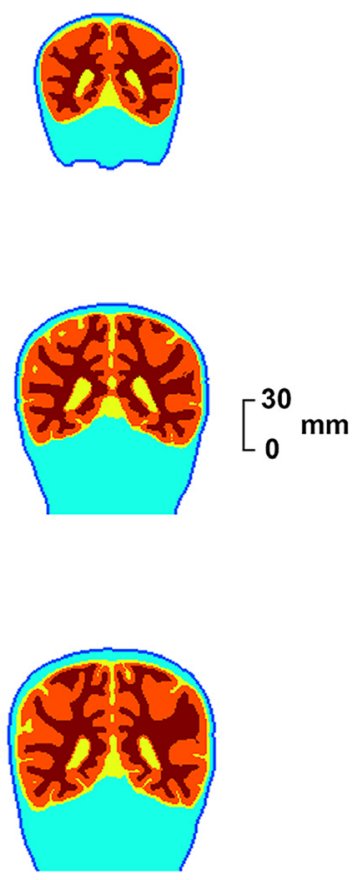

White matter

(c)

Fig. 1 Age-appropriate five-layered head models for (a) 0-yo; (b) 1-yo; and (c) 2-yo infants, which comprise the scalp, skull, CSF, GM, and WM. The first, second, and third columns show the sagittal, axial, and coronal views of the head models, respectively.

$$
q_{v o l}=\frac{12 \times(3 \times v o l)^{\frac{2}{3}}}{\sum_{0 \leq i \leq j \leq 3} l_{i, j}^{2}}
$$

where $v o l$ is the tetrahedral volume, and $l_{i, j}$ are the lengths of the edges of the tetrahedron. This metric is equal to 1 for equilateral tetrahedra and tends to zero for degenerated tetrahedra. The higher the $q_{v o l}$ value, the higher the quality of the mesh. In Table 1, we report the total number $(N)$ of nodes, elements, and faces. The mean $q_{v o l}$ (across all tetrahedrons) with its standard deviation is also shown. We also found the majority of volumetric tetrahedral meshes for each age group had a high Joe-Liu quality value $(76.9 \%, 80.5 \%$, and $79.2 \%$ of all meshes have JoeLiu quality values higher than 0.7 for 0 - to 2-yo infants). Light propagation in the head models was calculated using the Nirfast software, ${ }^{48}$ a finite element-based package that uses the diffusion approximation for modeling near-infrared light transport in tissue. ${ }^{49,50}$ The optical properties of each tissue type in the infant head models for a wavelength of $800 \mathrm{~nm}$ were specified as in

Table 1 Properties of the volumetric mesh (number of nodes, elements, faces, and the Joe-Liu quality index) for every age.

\begin{tabular}{lccc}
\hline \hline & 0-yo & 1-yo & 2-yo \\
\hline N nodes & 101056 & 212418 & 243541 \\
$N$ elements & 594241 & 1260896 & 1451619 \\
$N$ faces & 293208 & 519898 & 555656 \\
Mean $q_{\text {vol }} \pm$ std & $0.784 \pm 0.131$ & $0.799 \pm 0.123$ & $0.793 \pm 0.127$ \\
\hline \hline
\end{tabular}


Cai et al.: Correlating functional near-infrared spectroscopy with underlying cortical regions...

Table 2 Optical properties of tissue types for light propagation analysis.

\begin{tabular}{lcccc}
\hline \hline & $\mu_{a}\left(\mathrm{~mm}^{-1}\right)$ & $\mu_{s}\left(\mathrm{~mm}^{-1}\right)$ & $g$ & $n$ \\
\hline Scalp & 0.018 & 19 & 0.9 & 1.4 \\
Skull & 0.016 & 16 & 0.9 & 1.4 \\
CSF & 0.0041 & 0.32 & 0.9 & 1.4 \\
GM & 0.048 & 5.0 & 0.9 & 1.4 \\
WM & 0.037 & 10 & 0.9 & 1.4 \\
\hline \hline
\end{tabular}

previous studies, ${ }^{35,51}$ namely, the absorption coefficient $\mu_{a}$, scattering coefficient $\mu_{s}$, anisotropy factor g, and refractive index $n$ (Table 2). Notably, the reduced scattering coefficient $\mu_{s}{ }^{\prime}$ should be used to analyze light propagation using the diffusion equation, where $\mu_{s}{ }^{\prime}=\mu_{s}(1-g)$.

\subsubsection{Fiducial points and arrangement of $S D$ pairs}

The international 10-10 system positions were virtually set on the age-appropriate head models of 0-, 1-, and 2-yo using custom analysis scripts written in MATLAB (MathWorks, Natick, Massachusetts). First, we manually identified the four anatomical landmarks, including the inion, nasion, and left and right periauricular points on the scalp surface of the infant head models. Then, 61 fiducial points of the $10-10$ system $^{16,52}$ were automatically assigned to the scalp of the head model of every age, as depicted in Fig. 2.

Because the SD distance significantly affects the sampling regions, the distances between sources and detectors were set as $10,15,20,25$, and $30 \mathrm{~mm}$, where the midpoints of the SD pairs were set at the 10-10 fiducial points. Two SD pairs were placed according to the circumferential and vertical orientations at each fiducial point to examine whether the probe orientation influences the SCC for 0-, 1-, and 2-yo. The SD pair arrangements on the head models for five different $\mathrm{SD}$ distances for each age are shown in Fig. 3.
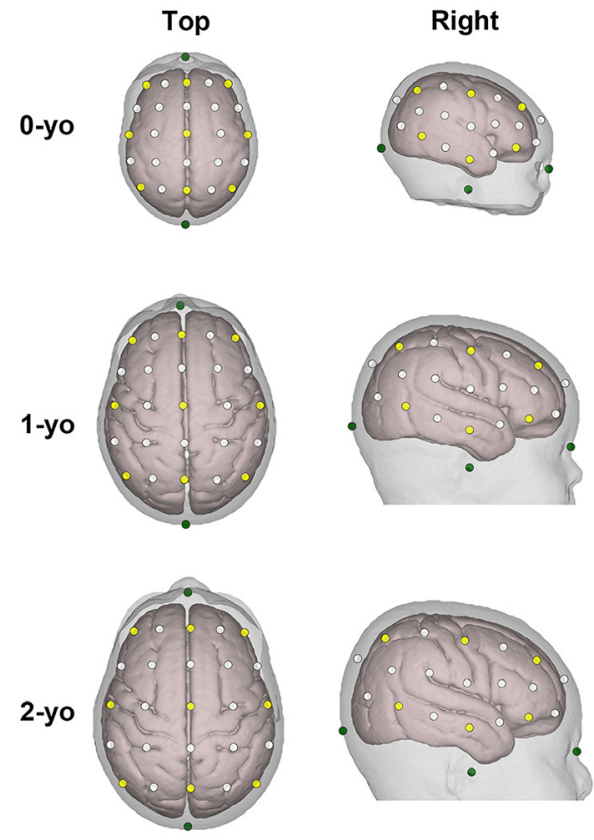

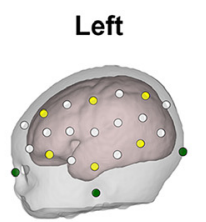

(a)
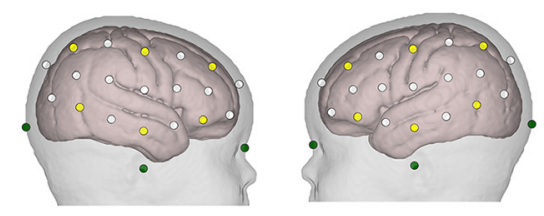

(b)

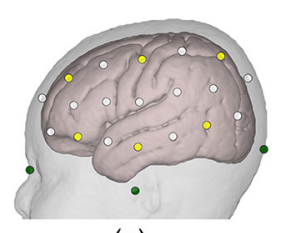

(c)

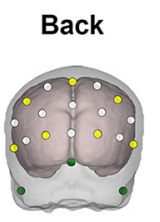

Front
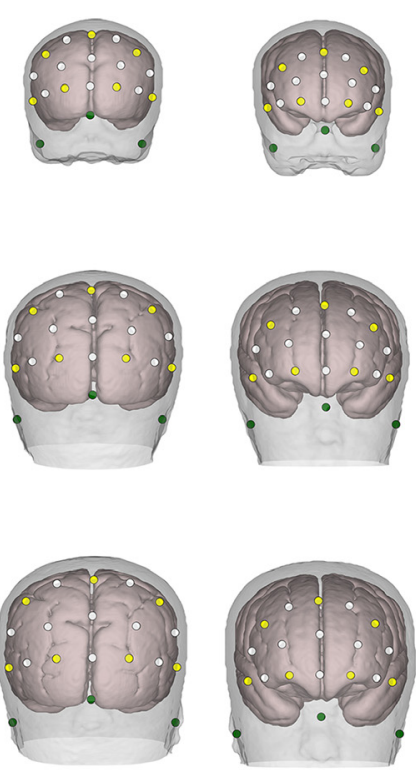

Fig. 2 Superimposed anatomical landmarks (in dark green), 10-20 fiducial points (in yellow), and 10-10 fiducial points (in white) on the head models of (a) 0-yo; (b) 1-yo; and (c) 2-yo. For each row panel, five views of the head models are displayed. 

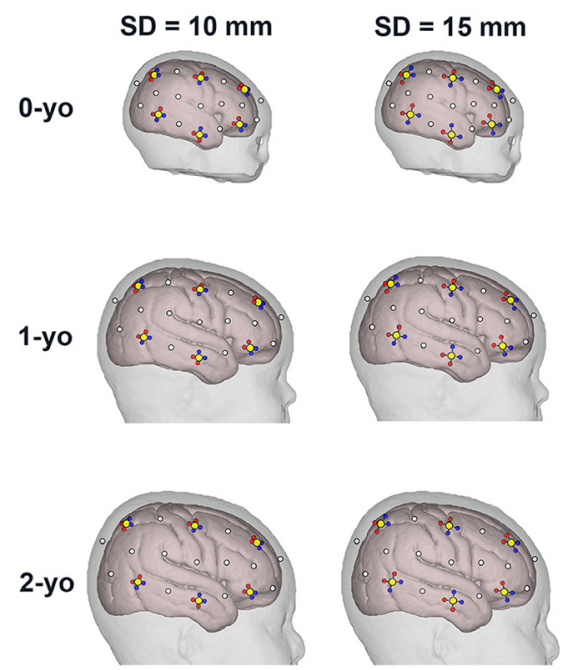
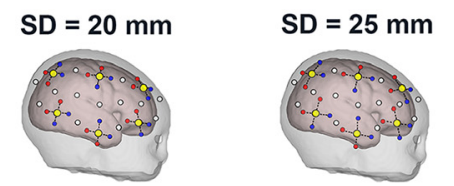

(a)

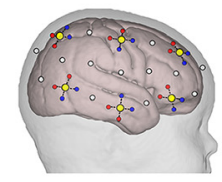

(b)

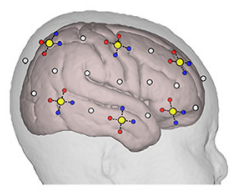

(c)

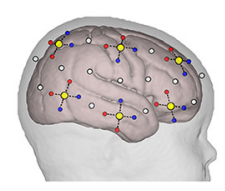

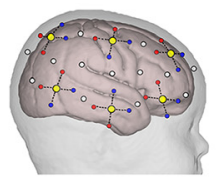
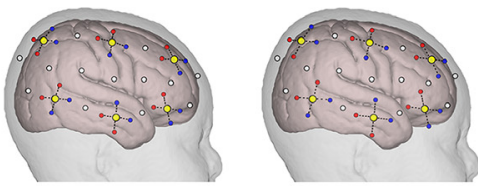

Fig. 3 SD pair arrangements on head models for five different SD distances for (a) 0-yo; (b) 1-yo; and (c) 2-yo. The sources and detectors are indicated in red and blue dots, respectively. Two orientational SD pairs at the 10-20 fiducial points (yellow dots) are displayed only on the right hemisphere. Notably, SD pairs were also attached to the 10-10 fiducial points (white dots); however, the sources and detectors at these locations are not shown to avoid complications. The circumferential and vertical SD pairs at each fiducial point are indicated by two dashed black connections, respectively.

\subsubsection{Calculation of photon measurement density function and normalized PPL}

Similar to our previous adult study, ${ }^{38}$ we adopted the optics-based method to analyze SCC. Based on the results of the light propagation analysis, the photon measurement density function (PMDF), which has the same spatial distribution as $\mathrm{SSP}^{53}$ was calculated at each node of the mesh model. ${ }^{54}$ As the sum of the SSPs is equivalent to the PPL in a particular brain region, the PMDF sum is linearly related to the PPL for that region. Given that each node in the mesh model was labeled as a specific AAL brain region, we could define the normalized PPL $\left(L_{n o r m, M}\right)$ in a given brain region $M$ using the following equation to quantify the SCC:

$$
L_{n o r m, M}=\frac{l_{M}}{\sum_{j=1}^{N} l_{j}},
$$

where $l_{M}$ and $l_{j}$ are the sum of the PMDFs of all nodes within the brain regions $M$ and $j$, respectively. $N$ is the total number of brain regions within the whole brain tissue. According to the equation, $L_{n o r m, M}$ ranges from 0 to 1 . Because the PMDF is a probability density function, ${ }^{55}$ we could use $L_{n o r m, M}$ as an index to represent the probability that the fNIRS signal is affected by the brain activation of region $M$. We calculated $L_{n o r m, M}$ of two orientational SD pairs (circumferential and vertical orientations) placed at every fiducial point for the 0-, 1-, and 2-yo head models.

\subsection{Characterization of SCC}

\subsubsection{Evaluation metrics}

For fNIRS users who are involved in developmental neuroscience, the following four questions are foremost: (1) how many brain regions are associated with an SD pair; (2) which brain region is the most likely corresponding brain region (MLCBR) for a given SD pair and its probability; (3) whether the sensitivity is sufficient to measure brain activity in the MLCBR, and (4) whether the MLCBR for the same scalp location is consistent across early development. Therefore, we defined four metrics, namely, the number of corresponding brain regions $\left(N_{C B R}\right)$, the selectivity 
of the MLCBR, the sensitivity of the MLCBR, and the consistency of the MLCBR across physical development, to further characterize the SCC and to systematically investigate the effects of age and SD distance on the SCC.

The detailed definitions of the four metrics are described below. Due to the strong scattering of near-infrared light passing through head tissues, a fiducial point usually projects to more than one brain region. Hence, we simply counted the number of brain regions that were correlated to a given fiducial point as the $N_{C B R}$. The current study used $L_{n o r m, M}=0.05$, as a threshold to calculate $N_{C B R}$. Moreover, we chose the brain region with the largest $L_{n o r m, M}$, as the MLCBR for a given fiducial point. $L_{n o r m, M L C B R}$ were defined as the metrics of selectivity at the fiducial point. For the sensitivity metric, we calculated the absolute PPL of MLCBR. The absolute PPL in the MLCBR was obtained using the following equation:

$$
L_{a b s, M L C B R}=\frac{\ln \left(I_{\text {base }} / I_{\text {pert }}\right)}{0.001 \mu_{a, M L C B R}},
$$

where $I_{\text {base }}$ and $I_{\text {pert }}$ are the detected intensities when the absorption coefficient of the MLCBR $\left(\triangle \mu_{a, M L C B R}\right)$ is the baseline and perturbed states $(0.1 \%$ increase), respectively. Finally, to examine whether the MLCBR at the same fiducial point was consistent from 0- to 2-yo, all fiducial points were classified into five categories according to consistency: (1) completely consistent, i.e., the MLCBR was the same for the brain region among the three ages; (2) consistent between 0 - and 1-yo; (3) consistent between 0 - and 2-yo; (4) consistent between 1- and 2-yo; and (5) inconsistent between any age groups.

\subsubsection{Statistical analysis}

Statistical analyses were performed using R. Almost all analyses were performed using $\mathrm{R}$ version 3.6.3 in R Studio Version 1.2.5033 (RStudio Team, 2019). The following packages were used for data manipulation, visualization, and statistical tests: dplyr v1.0.2, tidyverse v1.3.0, rstatix v0.6.0, magrittr v1.5, nparLD v2.1, and ggpubr v0.4.0. Only $N_{C B R}$ was assessed with R 3.5.1, using the nparLD package. The $N_{C B R}$, selectivity, and sensitivity values at each 10-10 fiducial point (61 in total) were treated as dependent variables. Provided that the dependent variable $N_{C B R}$ deviated from normality and equal variability, we applied a rank-based nonparametric mixed model statistical method, nparLD ${ }^{56}$ with an F1-LD-F1 design, to investigate the effects of age and SD distance on $N_{C B R}$. We reported the Wald-type statistic (WTS) to assess the statistical significance of age, SD distance, and their interaction. If the interaction or main effects were significant, comparisons between two conditions were conducted using the nonparametric Mann-Whitney U test (independent samples) or Wilcoxon signed-rank test (dependent samples), followed by the Bonferroni method for multiple comparison adjustment. For the dependent variables of sensitivity or selectivity, a mixed-design ANOVA was conducted to examine the effects of age and SD distance. Specifically, the sensitivity and selectivity were subjected to two two-way mixed ANOVAs with the SD distance $(10,15,20,25$, and $30 \mathrm{~mm})$ as a within-subjects factor and age $(0-, 1-$, and 2-yo) as a between-subjects factor. If the interaction or main effects were significant, comparisons between two conditions were conducted using a two-sample $t$-test (independent samples) or paired $t$-test (dependent samples), followed by the Bonferroni method for multiple comparison adjustment. In all ANOVA analyses, Greenhouse-Geisser corrections were applied on violation of the sphericity assumption. The generalized eta squared ${ }^{57,58}$ served as estimates of the effect sizes.

\section{Results}

\subsection{Optics-Based SCC for a Representative Fiducial Point}

To illustrate the changes in SSP due to SD distance and age, the PMDF of the circumferential SD pair at the fiducial point T4 is shown in Fig. 4. The spatial distribution of the PMDF broadened as the SD distance increased, regardless of age. The values of $L_{n o r m, M}$ higher than 0.01 , 
Cai et al.: Correlating functional near-infrared spectroscopy with underlying cortical regions...

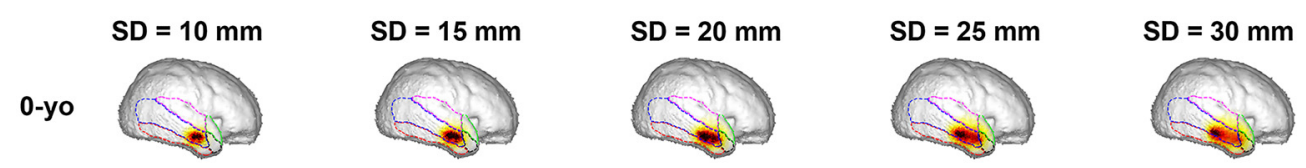

(a)
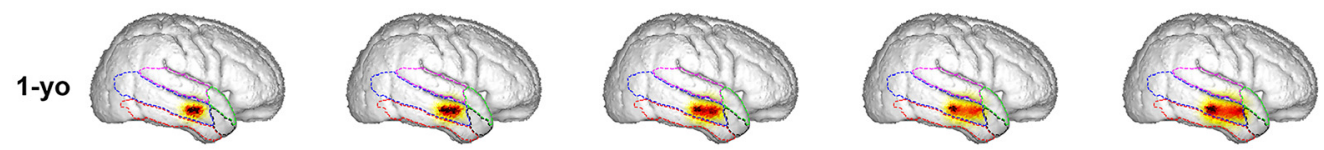

(b)
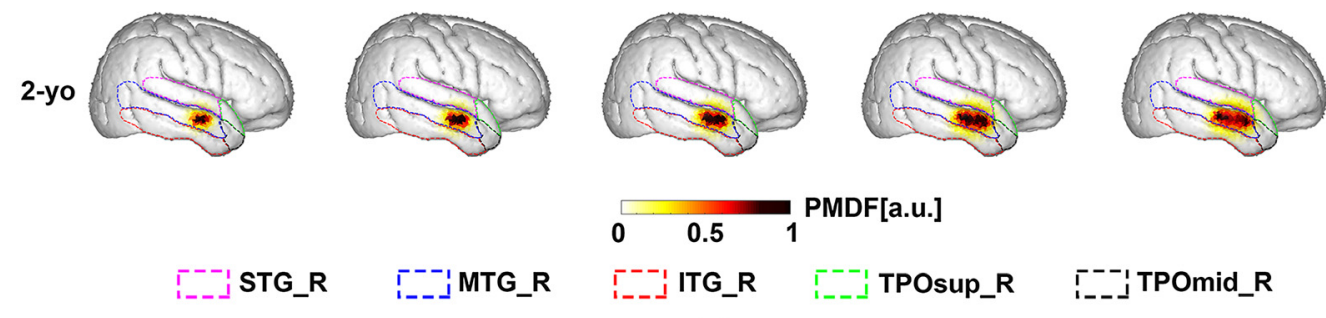

(c)

Fig. 4 PMDF for a given fiducial point T4 at five SD distances for 0-yo, 1-yo, and 2-yo when the SD pair was attached in a circumferential orientation. Dashed lines in different colors indicate the AAL brain region boundaries. The PMDF superimposed on age-appropriate brain structures of (a) 0-yo; (b) 1-yo; and (c) 2-yo.

at the fiducial point T4 are shown in Table 3. According to the definition of the $N_{C B R}$ (i.e., the number of brain regions whose $L_{n o r m, M}$ was $\left.>0.05\right), N_{C B R}$ was 2 at the fiducial point T4, which depicts the right middle and inferior temporal gyrus (MTG-R and ITG-R, respectively). These two brain regions had dominant $L_{n o r m, M}$ at the fiducial point T4 for any age and SD distance (indicated in boldface in Table 3), where the MLCBR was MTG_R. In addition to MTG_R and ITG_R, other brain regions, the superior temporal gyrus (STG-R) and right temporal pole of the superior and middle temporal gyrus (TPOsup-R and TPOmid-R, respectively), were also associated with $\mathrm{T} 4$, and their $L_{n o r m, M}$ increased as the SD distance increased. Considering the STG_R of 0-yo as an example, T4 had a $L_{n o r m, S T G_{-} R}$ of 0.009 for a $10-\mathrm{mm}$ SD distance, whereas the $L_{\text {norm,STG_R }}$ reached 0.022 when the SD distance was $30 \mathrm{~mm}$ (see Table 3). For the same SD distance, the $L_{\text {norm, } M T G_{-} R}$ of 1 - or 2 -yo was greater than that of 0 -yo. For instance, the $L_{n o r m, M T G_{-} R}$ values for a $20-\mathrm{mm}$ SD distance were $0.593,0.786$, and 0.861 for $0-, 1-$, and 2-yo, respectively. The PMDF of the vertical SD pair set at fiducial point T4 is shown in Fig. S1 in the Supplementary Material. Likewise, we found that the vertical SD pair at T4 was mainly correlated with the two brain regions, that is, MTG_R and ITG_R. The maximum $L_{n o r m, S T G_{-} R}$ of the vertical SD pair was only $0.060,0.070$, and 0.094 for 0-, 1-, and 2-yo, respectively ( $\mathrm{SD}$ distance $=30 \mathrm{~mm}$ ). As shown in Table 3, the fiducial point $\mathrm{T} 4$ had the same MLCBR, that is, MTG_R, for any age and SD distance; therefore, the MLCBR was completely consistent among the three ages. The sensitivity of $\operatorname{MLCBR}\left(L_{a b s, M L C B R}\right)$ is also shown in Table 3. We also observed an increase in the $L_{a b s, M L C B R}$ at each age with increasing SD distance. The $L_{a b s, M L C B R}$ of 0-yo was smaller than that of 1- and 2-yo, while $L_{a b s, M L C B R}$ of 1-yo was almost similar or slightly larger than that of 2-yo.

The brain regions correlated with the two orientational SD pairs at all 10-10 fiducial points and their normalized PPL from 0-, 1-, and 2-yo infants are provided in Tables S3-7 in the Supplementary Material for SD distances of 10, 15, 20, 25, and $30 \mathrm{~mm}$, respectively.

\subsection{Evaluation of SCC at All Fiducial Points}

The SCC metrics $N_{C B R}$, selectivity, and sensitivity at all 10-10 fiducial points are shown in Figs. 5-7, respectively. The summary statistics of the SCC metrics across all fiducial points are shown in Fig. 8. 
Table 3 Normalized PPL of corresponding brain regions $\left(L_{n o r m, M}\right)$ and the sensitivity of MLCBR $\left(L_{a b s, M L C B R}\right)$ for the circumferential SD pair set at the fiducial point T4 at five SD distances for 0-yo, 1-yo, and 2-yo.

\begin{tabular}{|c|c|c|c|c|c|c|}
\hline \multirow[b]{2}{*}{ Age } & \multirow[b]{2}{*}{ Brain region } & \multicolumn{5}{|c|}{ SD distance } \\
\hline & & $10 \mathrm{~mm}$ & $15 \mathrm{~mm}$ & $20 \mathrm{~mm}$ & $25 \mathrm{~mm}$ & $30 \mathrm{~mm}$ \\
\hline \multirow[t]{6}{*}{0 -yo } & MTG_R & 0.636 & 0.651 & 0.593 & 0.611 & 0.562 \\
\hline & ITG_R & 0.346 & 0.318 & 0.366 & 0.332 & 0.366 \\
\hline & STG_R & 0.009 & 0.013 & 0.014 & 0.021 & 0.022 \\
\hline & TPOsup_R & 0.004 & 0.007 & 0.009 & 0.013 & 0.017 \\
\hline & TPOmid_R & 0.003 & 0.006 & 0.010 & 0.013 & 0.020 \\
\hline & $L_{a b s, M L C B R}(\mathrm{~mm})$ & 1.9 & 4.6 & 5.9 & 7.9 & 9.1 \\
\hline \multirow[t]{6}{*}{ 1-yo } & MTG_R & 0.836 & 0.818 & 0.786 & 0.786 & 0.746 \\
\hline & ITG_R & 0.153 & 0.168 & 0.196 & 0.188 & 0.224 \\
\hline & STG_R & 0.009 & 0.011 & 0.014 & 0.020 & 0.022 \\
\hline & TPOsup_R & 0.001 & 0.001 & 0.001 & 0.002 & 0.003 \\
\hline & TPOmid_R & 0.000 & 0.000 & 0.001 & 0.001 & 0.002 \\
\hline & $L_{a b s, M L C B R}(\mathrm{~mm})$ & 4.6 & 6.2 & 8.0 & 10.4 & 11.3 \\
\hline \multirow[t]{6}{*}{ 2-yo } & MTG_R & 0.890 & 0.869 & 0.861 & 0.824 & 0.819 \\
\hline & ITG_R & 0.078 & 0.097 & 0.094 & 0.124 & 0.117 \\
\hline & STG_R & 0.026 & 0.027 & 0.034 & 0.037 & 0.046 \\
\hline & TPOsup_R & 0.002 & 0.003 & 0.004 & 0.007 & 0.009 \\
\hline & TPOmid_R & 0.001 & 0.001 & 0.001 & 0.003 & 0.003 \\
\hline & $L_{a b s, M L C B R}(\mathrm{~mm})$ & 3.9 & 5.8 & 7.6 & 9.8 & 11.3 \\
\hline
\end{tabular}

Note: The $L_{n o r m, M}$ value higher than 0.05 , is shown in bold. The MLCBR at T4 was MTG_R for all ages and SD distances.

We calculated the $N_{C B R}$ value for all 10-10 fiducial points over five SD distances at three ages. As shown in Fig. 5 for circumferential SD pairs, fiducial points neighboring the longitudinal fissure, for example, $\mathrm{Fpz}, \mathrm{FCz}, \mathrm{Cz}, \mathrm{CPz}$, and $\mathrm{POz}$, were correlated with more brain regions for every SD distance and age. Additionally, the larger the SD distance, the larger the $N_{C B R}$ of the fiducial points for every age group. The $N_{C B R}$ of 0 -yo was larger than that of 1- and 2-yo, while the $N_{C B R}$ of 1- and 2-yo was similar for each SD distance. According to a nonparametric statistical analysis, we found significant main effects of SD distance [WTS $(4)=195.88$, $p<0.001]$ and age $[\operatorname{WTS}(2)=10.37, p<0.01]$, but no interaction $[\mathrm{WTS}(8)=9.01$, $p=0.34$ ]. For the age factor [Figs. 8(a) and 8(d) upper panel], multiple comparisons with the Bonferroni adjustment showed that the $N_{C B R}$ of 0 -yo was larger than that of 1- and 2-yo ( $p<0.001$, corrected); however, no significant differences were found between 1- and 2-yo ( $p=0.92$, corrected). For the SD distance factor [Figs. 8(a) and 8(d) lower panel], multiple comparisons between $N_{C B R}$ of any two SD distances produced significantly different values, and the $N_{C B R}$ values of larger SD distances were greater than those of smaller SD distances ( $p<0.001$, corrected). Similar results for the $N_{C B R}$ of the vertical SD pairs are shown in Fig. S2 in the Supplementary Material.

The selectivity of the MLCBR shown in Fig. 6 shows the ratio of the PPL in the MLCBR to its sum in the 90 AAL brain region. In a sense, the selectivity represents the probability that the 


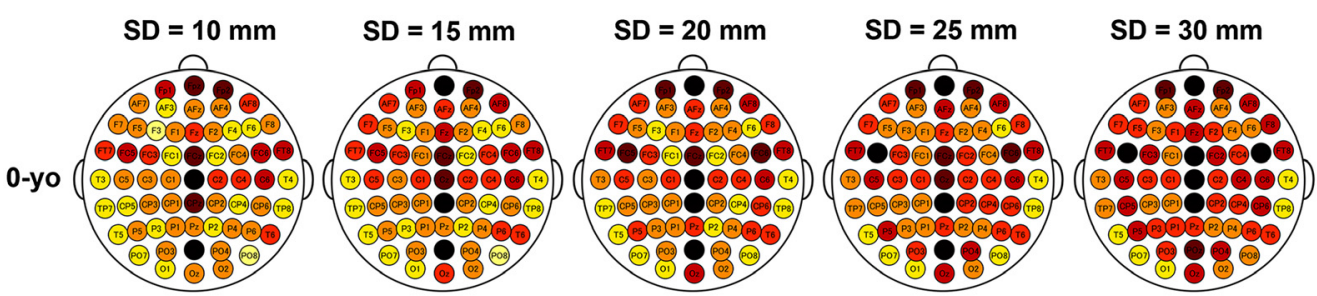

(a)
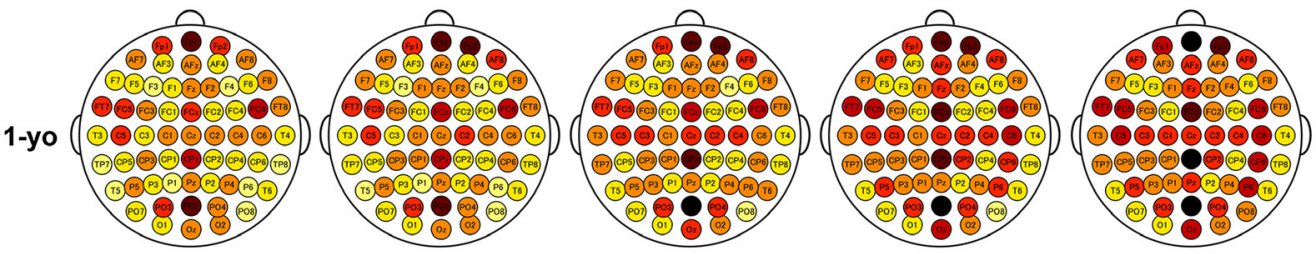

(b)
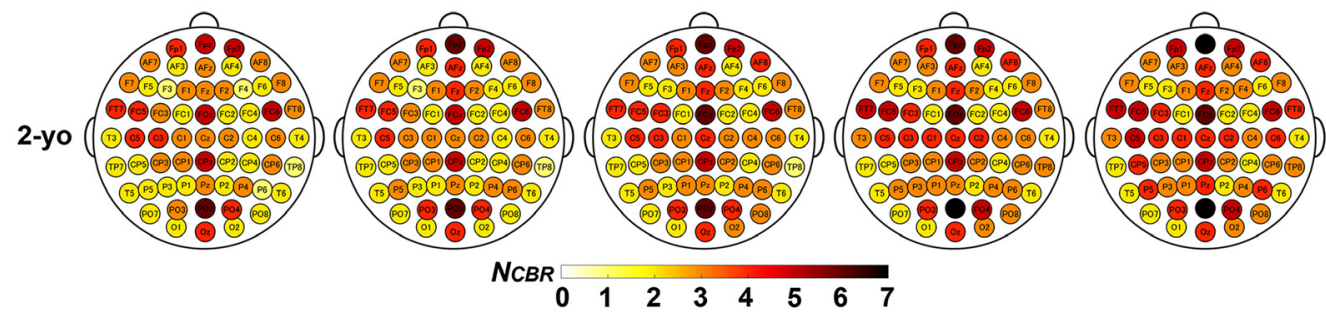

(c)

Fig. $5 N_{C B R}$ for all 10-10 fiducial points at five different SD distances for (a) 0-yo; (b) 1-yo; and (c) 2-yo. Darker red regions indicate larger $N_{C B R}$.

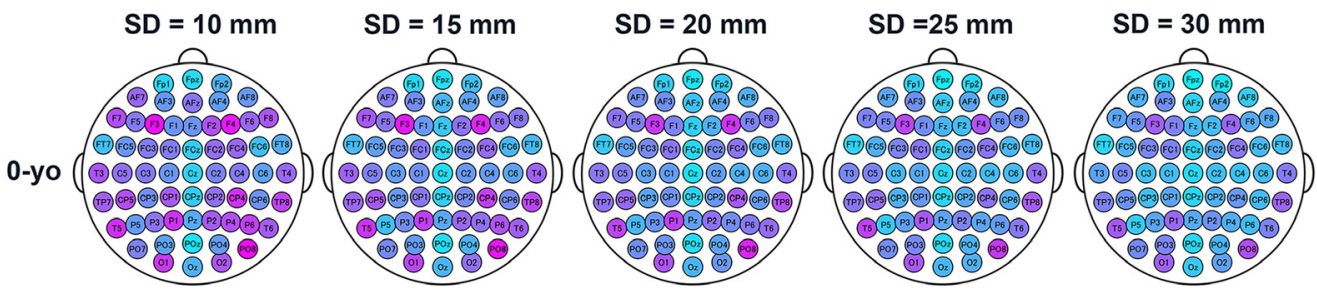

(a)
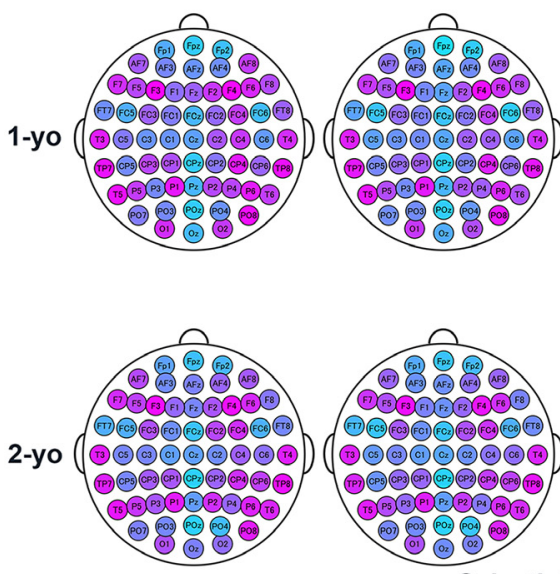

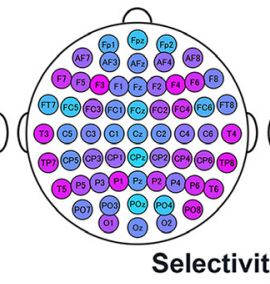

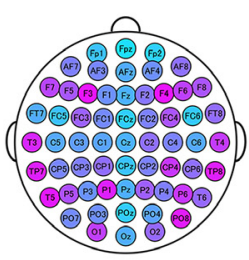

(b)
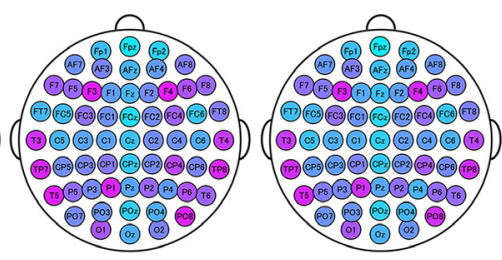
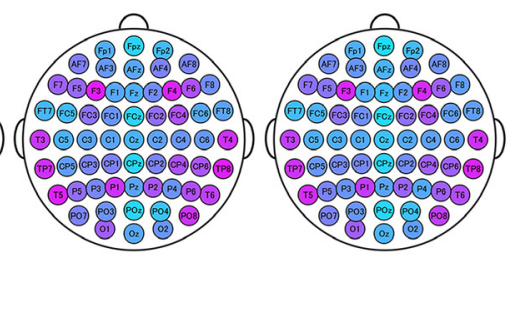

(c)

Fig. 6 Selectivity of MLCBR for all 10-10 fiducial points at five different SD distances for (a) 0-yo; (b) 1-yo; and (c) 2-yo. Areas in magenta and blue indicate higher and lower selectivity, respectively. 

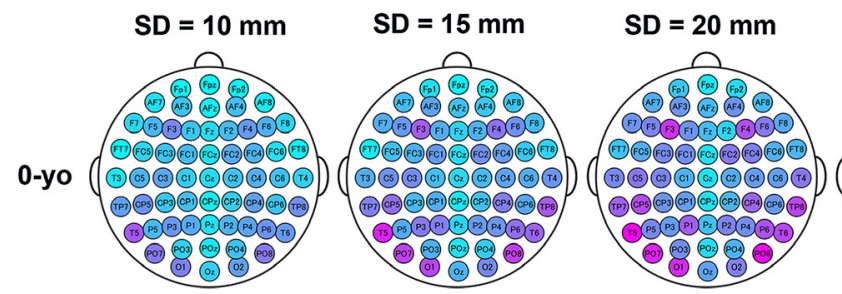

(a)
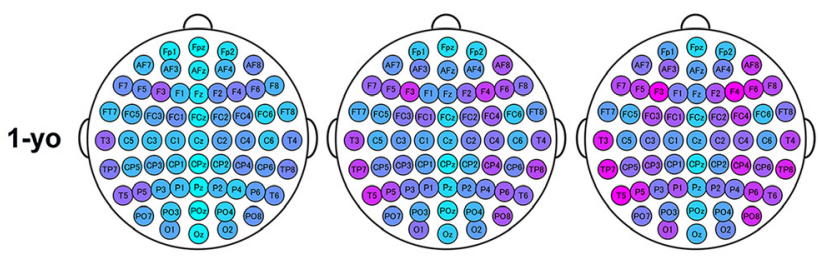

(b)
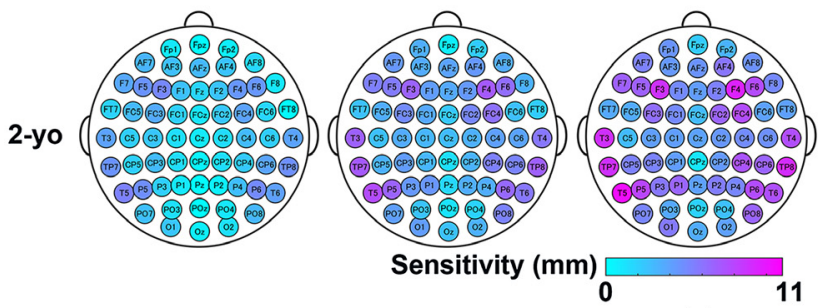

(c)
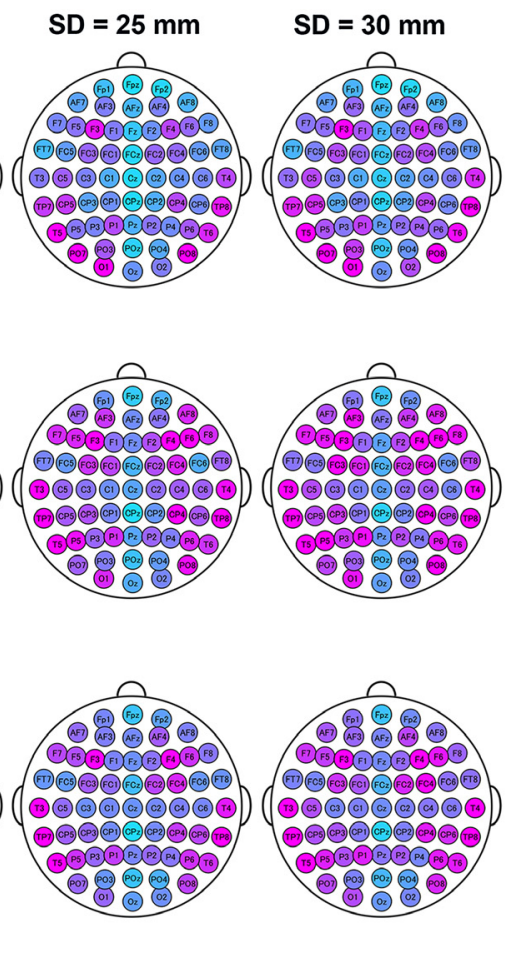

Fig. 7 Sensitivity of MLCBR for all 10-10 fiducial points at five different SD distances for (a) 0-yo; (b) 1-yo; and (c) 2-yo. Area in magenta and blue indicate higher and lower sensitivity, respectively.

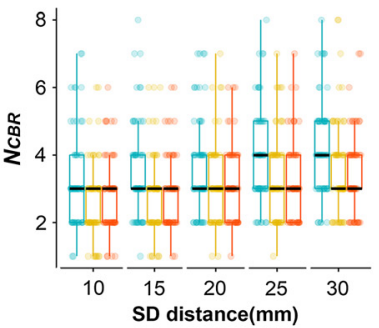

(a)
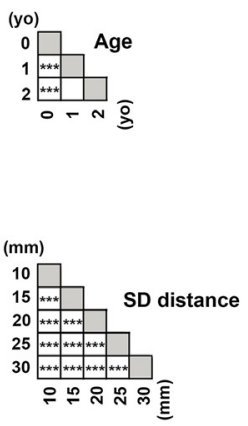

(d)

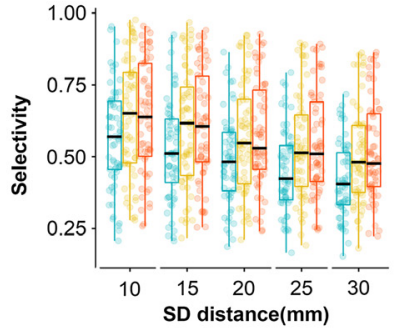

(b)
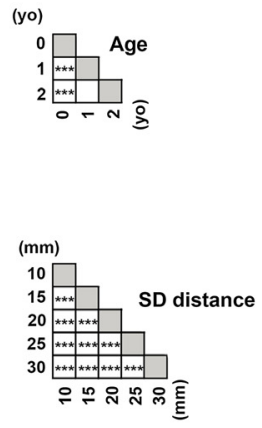

(e)

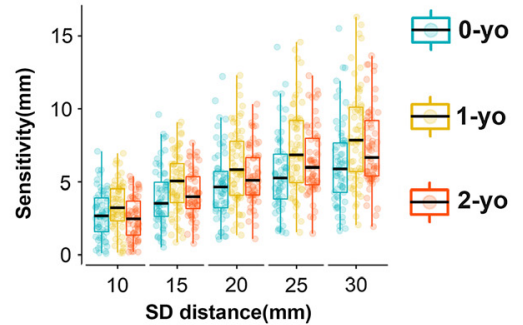

(c)

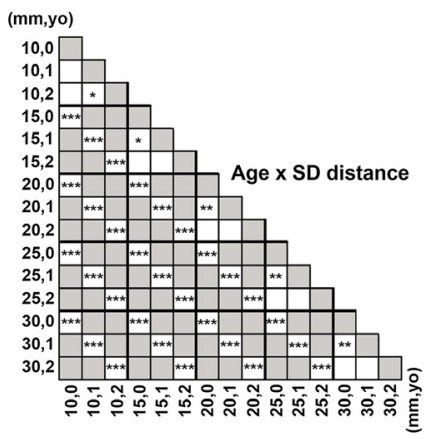

(f)

Fig. 8 Box plots summarizing the SCC metrics, (a) $N_{C B R}$; (b) selectivity; and (c) sensitivity, at all scalp fiducial points of the 10-10 system for each age and SD distance. The individual colored dots indicate the SCC metrics of each fiducial point. Boxes indicate the interquartile range. The black horizontal line within the boxes indicates the median. Whiskers extend 1.5 times above and below the interquartile range limits. Statistical significance of post-hoc test for (d) $N_{C B R}$; (e) selectivity; and (f) sensitivity are indicated with matrices. ${ }^{*} p<0.05,{ }^{* \star} p<0.01,{ }^{* * *} p<0.001$, white and gray blanks are not significant and not applicable, respectively. 
Cai et al.: Correlating functional near-infrared spectroscopy with underlying cortical regions...

fNIRS signal originates from the MLCBR. For most fiducial points, higher selectivity was observed for shorter SD distances, regardless of age. Moreover, the selectivity of 1- and 2 -yo was larger than that of 0 -yo. These observable findings were supported by statistically significant main effects of age $\left[\mathrm{F}(2,180)=4.89, p<0.05, \eta_{\mathrm{G}}{ }^{2}=0.049\right]$ and SD distance $\left[\mathrm{F}(1.41,253.25)=266.11, p<0.001, \eta_{\mathrm{G}}{ }^{2}=0.067\right]$ on selectivity; however, no statistically significant two-way interactions were found between age and SD distance on the selectivity, $\mathrm{F}(2.81,253.25)=0.93, p=0.42, \eta_{\mathrm{G}}{ }^{2}=0.001$ [Fig. 8(b)]. The results of the post-hoc multiple comparisons are shown in Fig. 8(e). For the age factor [Fig. 8(e), upper panel], multiple pairwise independent sample t-tests showed that the selectivity of 0-yo was lower than that of 1- and 2-yo for all fiducial points on average ( $p<0.001$, Bonferroni-corrected). In contrast, multiple pairwise paired $t$-tests for the SD distance [Fig. 8(e), lower panel] showed that comparisons from any two SD distances were significantly different $(p<0.001$, corrected) and that the selectivity decreased with increasing SD distance. Similar results for the selectivity of vertical SD pairs are shown in Fig. S3 in the Supplementary Material.

With regard to the sensitivity of the MLCBR over all 10-10 fiducial points, we found an obvious increase in $L_{a b s, M L C B R}$ with increasing SD distance for each age (Fig. 7). However, no differences in age were observed during the visual inspection. The 3 (age) $\times 5$ (SD distance) mixed ANOVA revealed significant main effects of age $\left[\mathrm{F}(2,180)=4.87, p<0.01, \eta_{\mathrm{G}}{ }^{2}=\right.$ $0.047]$ and SD distance $\left[\mathrm{F}(1.37,247.25)=837.02, p<0.001, \eta_{\mathrm{G}}{ }^{2}=0.306\right]$. However, these main effects were further qualified by the presence of a significant interaction between age and SD distance, $\mathrm{F}(2.75,247.25)=8.67, p<0.001, \eta_{\mathrm{G}}{ }^{2}=0.009$ [Fig. 8(c)]. The simple main effect of age was significant for all SD distances, that is, 10, 15, 20, 25, and $30 \mathrm{~mm}$ (all $p<0.05$ ). The results of the post-hoc multiple comparisons are shown in Fig. 8(f). At a 10-mm SD distance, the mean sensitivity of 1 -yo was significantly higher than that of 2-yo $(p<0.05$, Bonferronicorrected). The mean sensitivity of 1 -yo was significantly higher than that of 0 -yo at SD distances of $15 \mathrm{~mm}$ ( $p<0.05$, corrected), $20 \mathrm{~mm}(p<0.01$, corrected), $25 \mathrm{~mm}(p<0.01$, corrected), and $30 \mathrm{~mm}(p<0.01$, corrected). The simple main effect of the SD distance was also significant for any of the $0-, 1-$, and 2-yo infants (all $p<0.001$ ). At all years of age, the sensitivity of larger SD distances was significantly greater than that of smaller SD distances [Figs. 8(c) and 8(f), $p<0.001$, corrected]. Similar results for the sensitivity of the vertical SD pairs are presented in Fig. S4 in the Supplementary Material.

We found that almost half of 10-10 fiducial points correlated with the completely consistent MLCBR across the three ages for each SD distance (Fig. 9). The average number of fiducial points correlated with the completely consistent MLCBR for all SD distances was $41.6 \pm 1.5$ and $39.6 \pm 0.9$ for circumferential and vertical SD pairs, respectively, as shown in green circles in Fig. 9. However, fiducial points correlated with inconsistent MLCBR across the three ages (yellow and red circles) were found around the longitudinal fissure for both SD pair orientations. To help fNIRS researchers examine longitudinal functional development from 0 - to 2-yo, we have also provided fiducial points whose MLCBR was completely consistent at certain SD distances for the circumferential and vertical SD pairs across 0-, 1-, and 2-yo infants (Table 4).

\subsection{Suitable SD Distances for infant fNIRS Based on SCC Metrics}

Based on the results in Sec. 3.2, we found that the sensitivity and selectivity of the MLCBR increased and decreased, respectively, as the SD distance increased. In other words, there is a trade-off between the sensitivity and selectivity of the MLCBR in determining a suitable SD distance for targeting the brain regions of interest in infant fNIRS. Therefore, a suitable SD distance that achieves a good balance between the sensitivity and selectivity of the MLCBR in infant fNIRS was explored.

First, we drew a scatter plot of the selectivity and sensitivity for all 10-10 fiducial points at five SD distances at each infant age [Figs. 10(a)-10(c)]. Then, we empirically chose thresholds 3 and 0.4 for the sensitivity and the selectivity, respectively, to categorize every point in the scatter plot into three zones. Because sensitivity takes precedence over selectivity in fNIRS, we defined the red zone as an area with a sensitivity $<3$. The remaining region was divided into two zones based on the selectivity threshold. Fiducial points with a sensitivity lower and $>0.4$, were classified into yellow and green zones, respectively. Therefore, the SD distance with a larger number 


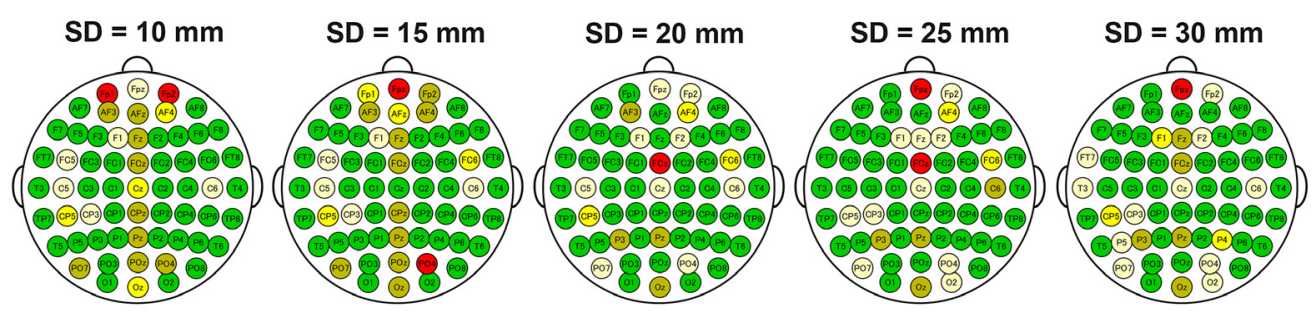

(a)
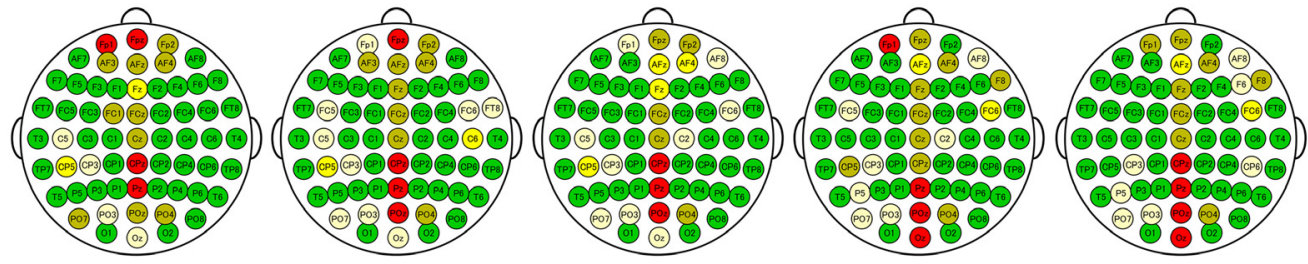

completely consistent

consistent between 0 -yo and 1-yo

consistent between 1-yo and 2-yo

consistent between 0 -yo and 2-yo

completely inconsistent

(b)

Fig. 9 Consistency of MLCBR at five SD distances for (a) circumferential and (b) vertical SD pairs. The consistency of the MLCBR among 0-yo, 1-yo, and 2-yo for every fiducial point is indicated by circles with different colors.

Table 4 Summary of fiducial points whose MLCBR was completely consistent at some SD distances for the circumferential and vertical SD pairs across 0-yo, 1-yo, and 2-yo infants.

\begin{tabular}{|c|c|c|c|c|}
\hline \multirow[b]{2}{*}{ Fiducial point } & \multicolumn{2}{|c|}{ Circumferential SD pairs } & \multicolumn{2}{|c|}{ Vertical SD pairs } \\
\hline & MLCBR & SD distance $(\mathrm{mm})$ & MLCBR & SD distance $(\mathrm{mm})$ \\
\hline $\mathrm{Cz}$ & SMA-R & 15 & - & - \\
\hline $\mathrm{AFz}$ & SFGmed-R & $20,25,30$ & - & - \\
\hline $\mathrm{Fz}$ & SFGmed-R & 20 & - & - \\
\hline $\mathrm{CPz}$ & PoCG-L & $20,25,30$ & - & - \\
\hline $\mathrm{POz}$ & SPG-L & $20,25,30$ & - & - \\
\hline T3 & MTG-L & $10,15,20,25$ & MTG-L & $10,15,20,25,30$ \\
\hline C5 & STG-L & 25,30 & PoCG-L & 25,30 \\
\hline C3 & PoCG-L & $10,15,20,25,30$ & PoCG-L & $10,15,20,25,30$ \\
\hline $\mathrm{C} 1$ & PreCG-L & $10,15,20,25,30$ & PreCG-L & $10,15,20,25,30$ \\
\hline $\mathrm{C} 2$ & PreCG-R & $10,15,20,25,30$ & PreCG-R & $10,15,30$ \\
\hline $\mathrm{C} 4$ & PoCG-R & $10,15,20,25,30$ & PoCG-R & $10,15,20,25,30$ \\
\hline C6 & - & - & PoCG-R & $10,20,25,30$ \\
\hline $\mathrm{T} 4$ & MTG-R & $10,15,20,25,30$ & MTG-R & $10,15,20,25,30$ \\
\hline FT7 & TPOsup-L & $10,15,20,25$ & TPOsup-L & $10,15,20,25,30$ \\
\hline F7 & ORBinf-L & $10,15,20,25,30$ & ORBinf-L & $10,15,20,25,30$ \\
\hline AF7 & ORBmid-L & $10,15,20,25,30$ & ORBmid-L & $10,15,20,25,30$ \\
\hline Fp1 & ORBmid-L & $20,25,30$ & - & - \\
\hline
\end{tabular}


Cai et al.: Correlating functional near-infrared spectroscopy with underlying cortical regions...

Table 4 (Continued).

\begin{tabular}{|c|c|c|c|c|}
\hline \multirow[b]{2}{*}{ Fiducial point } & \multicolumn{2}{|c|}{ Circumferential SD pairs } & \multicolumn{2}{|c|}{ Vertical SD pairs } \\
\hline & MLCBR & SD distance $(\mathrm{mm})$ & MLCBR & $\mathrm{SD}$ distance $(\mathrm{mm})$ \\
\hline Fp2 & - & - & SFGdor-R & 25,30 \\
\hline AF8 & ORBmid-R & $10,15,20,25,30$ & ORBmid-R & 10,15 \\
\hline F8 & ORBinf-R & $10,15,20,25,30$ & ORBinf-R & $10,15,20$ \\
\hline FT8 & MTG-R & $10,15,20,25,30$ & MTG-R & $10,20,25,30$ \\
\hline TP7 & MTG-L & $10,15,20,25,30$ & MTG-L & $10,15,20,25,30$ \\
\hline T5 & MTG-L & $10,15,20,25,30$ & MTG-L & $10,15,20,25,30$ \\
\hline 01 & MOG-L & $10,15,20,25,30$ & MOG-L & $10,15,20,25,30$ \\
\hline $\mathrm{O} 2$ & SOG-R & $10,15,20$ & SOG-R & $10,15,20,25,30$ \\
\hline PO8 & MOG-R & $10,15,20,25,30$ & MOG-R & $10,15,20,25,30$ \\
\hline T6 & MTG-R & $10,15,20,25,30$ & MTG-R & $10,15,20,25,30$ \\
\hline TP8 & MTG-R & $10,15,20,25,30$ & MTG-R & $10,15,20,25,30$ \\
\hline $\mathrm{FC5}$ & IFGtriang-L & $20,25,30$ & IFGoperc-L & $10,20,30$ \\
\hline $\mathrm{FC} 3$ & MFG-L & $10,15,20,25,30$ & MFG-L & $10,15,20,25,30$ \\
\hline $\mathrm{FC} 1$ & MFG-L & $10,15,20,25,30$ & MFG-L & $15,20,25,30$ \\
\hline $\mathrm{FC} 2$ & MFG-R & $10,15,20,25,30$ & MFG-R & $10,15,20,25,30$ \\
\hline $\mathrm{FC} 4$ & MFG-R & $10,15,20,25,30$ & MFG-R & $10,15,20,25,30$ \\
\hline FC6 & IFGoperc-R & 10,30 & IFGoperc-R & 10 \\
\hline F5 & IFGtriang-L & $10,15,20,25,30$ & IFGtriang-L & $10,15,20,25,30$ \\
\hline F3 & MFG-L & $10,15,20,25,30$ & MFG-L & $10,15,20,25,30$ \\
\hline $\mathrm{F} 1$ & - & - & SFGdor-L & $10,15,20,25,30$ \\
\hline $\mathrm{F} 2$ & SFGdor-R & 10,15 & SFGdor-R & $10,15,20,25,30$ \\
\hline $\mathrm{F} 4$ & MFG-R & $10,15,20,25,30$ & MFG-R & $10,15,20,25,30$ \\
\hline F6 & IFGtriang-R & $10,15,20,25,30$ & IFGtriang-R & $10,15,20,25$ \\
\hline AF3 & MFG-L & 25,30 & MFG-L & $20,25,30$ \\
\hline AF4 & MFG-R & 30 & - & - \\
\hline CP3 & IPL-L & 20 & - & - \\
\hline CP1 & PoCG-L & $10,15,20,25,30$ & PoCG-L & $10,15,20,25,30$ \\
\hline CP2 & PoCG-R & $10,15,20,25,30$ & PoCG-R & $10,15,20,25,30$ \\
\hline $\mathrm{CP} 4$ & IPL-R & $10,15,20,25,30$ & IPL-R & $10,15,20,25,30$ \\
\hline CP6 & SMG-R & $10,15,20,25,30$ & SMG-R & $10,15,20,25$ \\
\hline P5 & ANG-L & $10,15,20,25$ & ANG-L & $10,15,20$ \\
\hline P3 & ANG-L & 10,15 & ANG-L & $10,15,20,25,30$ \\
\hline P1 & SPG-L & $10,15,20,25,30$ & SPG-L & $10,15,20,25,30$ \\
\hline
\end{tabular}


Cai et al.: Correlating functional near-infrared spectroscopy with underlying cortical regions...

Table 4 (Continued).

\begin{tabular}{llclll}
\hline \hline & \multicolumn{2}{c}{ Circumferential SD pairs } & & \multicolumn{2}{c}{ Vertical SD pairs } \\
\cline { 2 - 3 } \cline { 5 - 6 } Fiducial point & MLCBR & SD distance $(\mathrm{mm})$ & & MLCBR & SD distance (mm) \\
\hline P2 & SPG-R & $10,15,20,25,30$ & & SPG-R & $10,15,20,25,30$ \\
P4 & ANG-R & $10,15,20,25$ & & ANG-R & $10,15,20,25,30$ \\
P6 & ANG-R & $10,15,20,25,30$ & & ANG-R & $10,15,20,25,30$ \\
PO3 & ANG-L & $10,15,20,25,30$ & & - & - \\
\hline
\end{tabular}

Note: the line '-' indicates that the category is not applicable. See Table S2 in the Supplementary Material for abbreviations of brain regions.

of fiducial points categorized into the green zone indicates that both higher sensitivity and selectivity can be achieved.

As shown in Fig. 10, the distribution of points in the scatter plot varies with the SD distance for infants at all ages [Figs. 10(a)-10(c) for 0-, 1-, and 2-yo infants, respectively]. For shorter and

0-yo
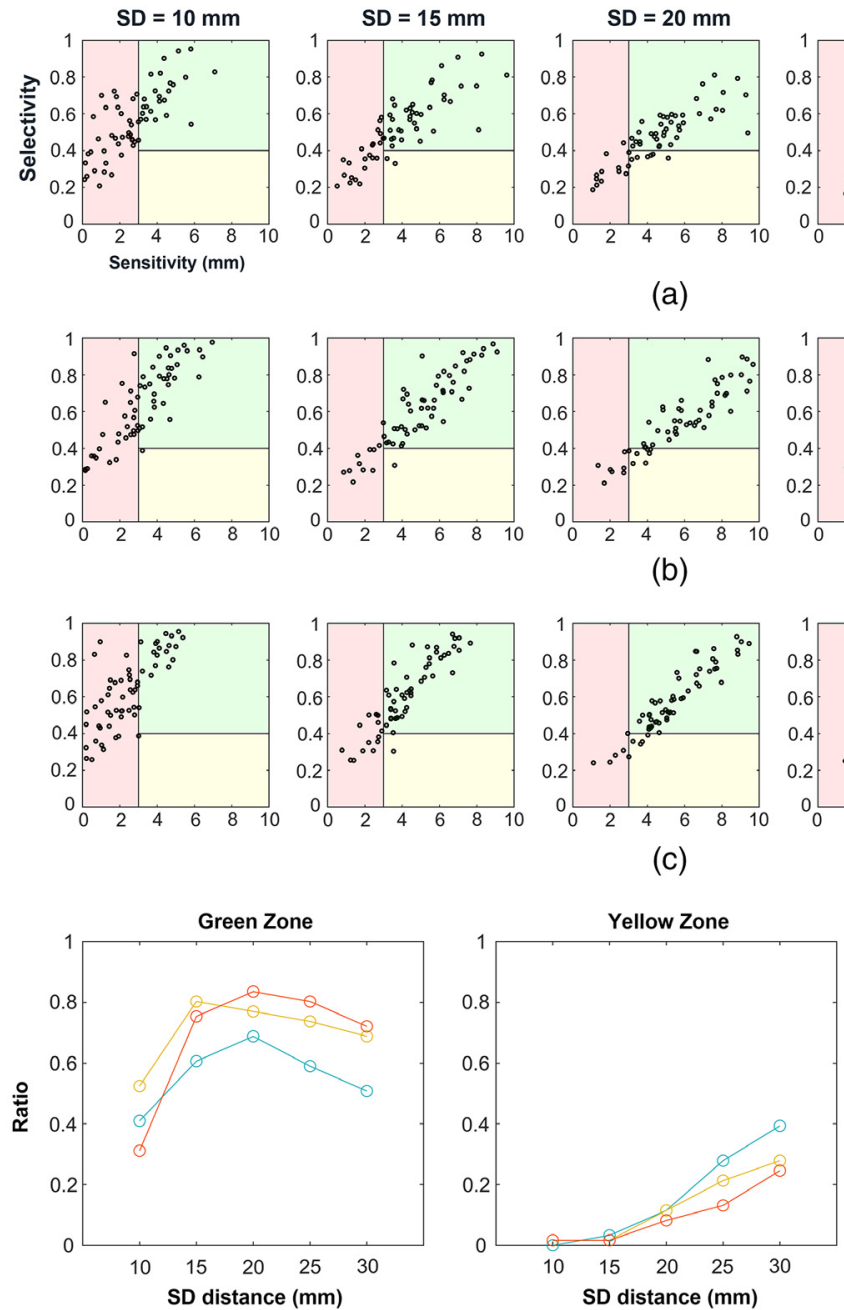

(d)
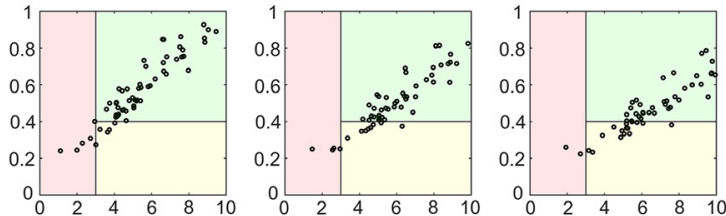

(c)
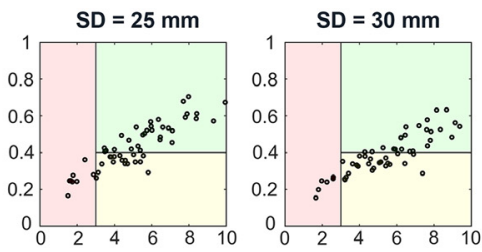

(a)
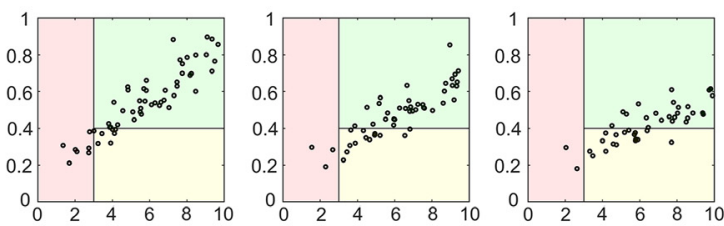

(b)

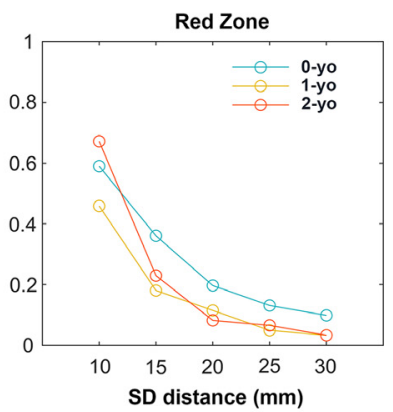

SD distance $(\mathrm{mm})$

Fig. 10 Relation between sensitivity and selectivity for each SD distance in (a) 0-yo; (b) 1-yo; and (c) 2-yo. Black circles in figures (a)-(c) indicate data from each 10-10 fiducial point. (d) Ratios of the number of fiducial points in the green, yellow, and red zones to the number of all 10-10 fiducial points. 
Cai et al.: Correlating functional near-infrared spectroscopy with underlying cortical regions...

longer SD distances, more fiducial points were observed in the red and yellow zones, respectively. The ratios of the number of fiducial points in each zone to all fiducial points are shown in Fig. 10(d). For the green zone, inverted U-shaped curves were observed for all three age groups. On the other hand, the ratio of fiducial points in the yellow and red zones showed a monotonic increase and decrease as the SD distance increased. These findings suggest that excessive short and long SD distances are unsuitable for infant fNIRS. Therefore, the SD distance between $15 \mathrm{~mm}$ and $25 \mathrm{~mm}$ could be suitable for 0-, 1-, and 2-yo infants. A $25 \mathrm{~mm}$ SD distance was more suitable if the sensitivity was emphasized, whereas a 15-mm SD distance was more appropriate if the selectivity was considered as a priority. A relatively balanced trade-off can be obtained when the SD distance is $20 \mathrm{~mm}$. To validate the robustness of this finding, we examined the same problem with different thresholds 4 and 0.5 for sensitivity and selectivity, respectively. Although the thresholds changed modestly, the shapes of the graph, as shown in Fig. 10(d), were maintained (Fig. S5 in the Supplementary Material).

\section{Discussion}

fNIRS has contributed significantly to the advancement of developmental cognitive neuroscience; however, fNIRS data cannot provide any anatomical brain information, which is critical for data explanation and comparisons with other modalities. Although several methods have been proposed to obtain SCC in adults and infants, little is known about the influence of age and SD distance on SCC with substantial physical development of the infant's head during the first two postnatal years. In the present study, we adopted light propagation analysis to establish a precise optics-based SCC between the fNIRS measurement channels, that is, SD pairs, set on the 10-10 system scalp positions and AAL brain regions in three age-appropriate head models of 0-, 1-, and 2-yo. Importantly, we provided four metrics: $N_{C B R}$, and the selectivity, sensitivity, and consistency of the MLCBR, to quantitatively evaluate the SCC for changes during a remarkable period of brain development. Moreover, we assessed the suitable SD distances for infant fNIRS by simultaneously considering the selectivity and sensitivity of the MLCBR.

\subsection{Optics-Based SCC Derived from Age-Appropriate Sophisticated Head Models}

Several studies have taken an important step toward the establishment of infant SCC; ${ }^{22-25}$ however, they only considered the head size of infants based on the simplified fNIRS principle that the signal comes from the cortical projection point below the midpoint of the SD pair. In fact, an fNIRS channel measures absorption changes in a broad cortical area, rather than at a single point. Thus, to establish a precise optics-based SCC that reflects light diffusion in the brains of 0-, 1-, and 2-yo infants, the key step is to construct sophisticated realistic head models that comprise multiple biological tissues with distinct optical properties such as scalp, skull, CSF, and brain tissues.

In this study, we constructed a five-layered head model for each age, distinguishable from a four-layered model where the skull and scalp constitute a single extracerebral layer. ${ }^{36,46,59}$ Separating the scalp and skull in infantile light propagation analysis could guarantee more accurate SCC compared with using the four-layered model, as these two types of infant tissues have their own optical properties ${ }^{35,51}$ and physical development. ${ }^{43,44}$

In addition, we employed template-based head models for light propagation analyses, because the acquisition of subject-specific MRI is generally challenging owing to the difficulty in controlling the motion of infants and undermines the intrinsic advantage of the fNIRS technique for facilitating functional brain measurements during early development. Notably, previous adult or infant studies have shown that the use of a template-based head model is useful for identifying the activation focus, although there are anatomical differences between subject-specific and template-based head models. ${ }^{24,25,60-62}$ Furthermore, the template-based model used in this study was derived from anatomical images of 95 healthy infants and reflects the general anatomical structure of infants. Therefore, the optics-based SCC obtained by analyzing light propagation in template-based infant head models of 0-, 1-, and 2-yo could provide a reliable database to guide fNIRS users in designing probe geometry, and to explain fNIRS data. 
Light propagation analysis depends on the optical properties of head tissues. In this study, the same optical properties of the neonate were assigned to all three age models because no established optical properties of head tissues were available for each age group in this study. On the other hand, optical properties in these ages may change due to alterations in tissue composition, such as myelination and bone mineral density during the first 2 years of life. As the level of bone mineral density was almost constant during the first year ${ }^{63}$ and slightly increased in the second year, ${ }^{64}$ the optical properties of the skull may not change during those years. In contrast, a substantial amount of myelination occurs in a wide range of brains. ${ }^{65}$ Thus, the alteration of the optical properties of WM and GM will definitely be at a non-negligible level. Light propagation is affected by differences in optical properties between adults and infants. ${ }^{35}$ To address the effect of changes in optical properties on the SCC, we attempted to use the optical properties of the GM and WM of adults to calculate the SCC for the oldest 2-yo head model. Compared to the results with infants' optical properties, additional simulation results revealed that the optical properties of adults applied to 2-yo had little effect on the SCC. Although a slightly broadened SSP was observed owing to the stronger scattering (Fig. S6 in the Supplementary Material for comparison between the optical properties of the neonate and adult), the consistency of the MLCBR among 0-, 1-, and 2-yo was unaffected by the difference in the optical properties (see Fig. S7 and Table S8 in the Supplementary Material for the optical properties of the adults). The actual changes in optical properties due to development from 0- to 2-yo would be smaller than those assumed here for neonates to adults. Therefore, we claim that our findings using the same optical properties for all three age groups could be used as a reference for developmental cognitive neuroscience. Meanwhile, the accuracy of optics-based SCC will be improved with the advance of in vivo measurement techniques for characterizing the optical properties of tissues. ${ }^{66}$ However, the measurement of accurate optical properties in vivo is still a challenging issue, as shown by the wide range of values reported by measurement techniques. ${ }^{36}$

\subsection{Effect of SD Distance and Physical Development on the SCC}

For a given fiducial point, the SSP was found to be a function of the SD distance, that is, the spatial range of the SSP broadened as the SD distance increased. This finding was consistent with previous studies, regardless of slab models ${ }^{30,67}$ or realistic head models ${ }^{68}$ being utilized. Intriguingly, the spatial distribution of the SSP values was also affected by age for a consistent SD distance (Fig. 4). Age-related alterations in SSP may be caused by changes in the local anatomical structures across the three age groups.

The optics-based SCC results (Table 4 and Table. S3-7 in the Supplementary Material) demonstrate that fNIRS signals interrogated by an SD pair always originate from multiple brain regions, even if the SD distance is $10 \mathrm{~mm}$. The one-to-many relationship between an SD pair and cortical regions is due to strong light scattering in the head tissue. In addition, we found several aspects related to $N_{C B R}$. As for the spatial characteristics, fiducial points around the longitudinal fissure were correlated with more brain regions than other points (Fig. 5), which may be because the cortices around the longitudinal fissure were parcellated into a relatively large number of regions in the AAL atlas. From the perspective of age-related change, we found that although the $N_{C B R}$ of 0 -yo was larger than that of 1- and 2-yo, there were no significant differences between the $N_{C B R}$ values of 1-o and 2-yo [Fig. 8(d)], which suggests that the similar spatial range of SSP for the same SD distance will occupy fewer brain regions when the head becomes larger owing to the physical development of the infant. In terms of the dependency on the SD distance, the finding that a larger SD distance had more corresponding brain regions [Figs. 5, 8(a), and 8(d)] could be clearly explained by the wider SSP at larger SD distances, as shown in Fig. 4.

We found that the selectivity of MLCBR decreased with increasing SD distance [Figs. 6 and 8(b)]. As mentioned before, the larger the SD distance, the wider the spatial range of the SSP in the brain. Hence, the increase in the normalized PPL of brain regions other than the MLCBR resulted in a relative decrease in the normalized PPL of the MLCBR. In addition, the selectivity of 1- and 2-yo was higher than that of 0 -yo on average for all fiducial points (Fig. 6). The difference in the physical development of the head in each year would have determined the degree of age-dependent change in the normalized PPL of the MLCBR. The 0-yo head size is obviously 
Cai et al.: Correlating functional near-infrared spectroscopy with underlying cortical regions...

smaller than that of 1- and 2-yo, while 1- and 2-yo have an almost similar head size (see Fig. 1 for example). In other words, the constant area of the brain surface is occupied by a small number of brain regions in the large brains of 1- and 2-yo, but by a large number in the small brains of 0-yo. Furthermore, the spatial range of the SSP was almost constant for the same SD distance, regardless of age. Thus, the constant spatial range of SSP across age was mainly occupied by the MLCBR in the larger brains of 1- and 2-yo, but distributed across several brain regions in the smaller brain of the 0-yo. Therefore, the normalized PPL in the MLCBR of the 0-yo infants was significantly smaller than that of the 1- and 2-yo infants.

We found that the sensitivity of the MLCBR significantly increased as the SD distance increased from 10 to $30 \mathrm{~mm}$ at 5-mm intervals for each age [Fig. 8(f)]. This finding is in accordance with evidence obtained from the change in GM sensitivity as a function of SD distance in the neonatal and adult head models. ${ }^{35}$ The sensitivity of 1-yo was significantly higher than that of 0 -yo at 15-, 20-, 25-, and 30-mm SD distances; however, the sensitivity of 2-yo was significantly lower than that of 1-yo at a $10 \mathrm{~mm} \mathrm{SD}$ distance [Fig. 8(f)]. One possible explanation for the increase or decrease in the sensitivity depending on the time of growth is that physical development differs between brain tissue and superficial layers such as the scalp and skull; the increase in the sensitivity from 0 - to 1-yo may be caused by the considerable expansion of the cortical surface during this period. Conversely, from 1- to 2-yo, the decrease in sensitivity may be due to relatively smaller expansion of the cortical surface, although the thickness of the superficial layers continues to increase.

We also found that the MLCBR was completely consistent at almost half of 10-10 fiducial points of 0-, 1-, and 2-yo for each SD distance. In other words, almost half of the fiducial points were correlated with one specific brain region with the largest normalized PPL (Fig. 9 and Table 4). This finding indicates that we can measure identical MLCBRs in longitudinal studies from 0- to 2-yo with fNIRS without the subject's own structural MRI when a probe of the same SD distance is attached at the scalp fiducial points. Thus, this finding is extremely uplifting for fNIRS users who are interested in the functional development of such MLCBRs. On the other hand, the MLCBR corresponding to fiducial points just above the longitudinal fissure was mostly inconsistent across the three ages (Fig. 9). The $N_{C B R}$ and $L_{n o r m, M L C B R}$ of the fiducial points were relatively larger and smaller than those of the other points, respectively (Figs. 5 and 6), which suggests that the MLCBR at a certain age may easily be replaced by another brain region at another age because of the slight change in the light path. One possibility for age-related changes in the light path is that the thickness of the skull in the frontal, parietal, and occipital regions along with the longitudinal fissure changes more drastically than in other locations from 0 - to 2 -yo. ${ }^{44}$ Another possibility is that the cortical surface expands relatively more in regions of the superior parietal, prefrontal, occipital cortices, and postcentral gyrus than in other regions during the first two years. ${ }^{2}$

The consistency of the MLCBR revealed in the present study could provide strong support for the reliability of findings from longitudinal developmental studies using fNIRS in which the probe was attached based on scalp fiducial points. In recent years, by executing the longitudinal cohort study projects [e.g., Brain Imaging for Global Health (BRIGHT) project], several laboratories worldwide have devoted themselves to investigating functional brain development during the first two postnatal years, including studies of visual working memory, ${ }^{69}$ social cognition, ${ }^{70,71}$ and resting-state functional networks. ${ }^{72}$ The present study will be especially useful for a large cohort of longitudinal or cross-sectional studies of early brain development, as structural MRI scanning, which is a demanding task for young populations, is not necessarily required.

\subsection{Consideration of Suitable SD Distances for Infant fNIRS}

The suitable SD distance for infants remains a matter of debate, as its choice is dependent on several factors. By considering a trade-off between the selectivity and sensitivity of the MLCBR, this study provided recommendations on suitable SD distances for 0-, 1-, and 2-yo. The most desirable SD distance is the one that has both the maximum number of fiducial points with high selectivity and sensitivity, and a minimum number of fiducial points with low selectivity and sensitivity. Based on this perspective, we determined that the suitable SD distances in fNIRS for infants during the first 2 years ranged from 15 to $25 \mathrm{~mm}$. This finding supports the results of a 
previous study in which the highest sensitivity was obtained at an SD distance of $20 \mathrm{~mm}$ in 3-month-old infants. ${ }^{39}$ In practical applications, we suggest that fNIRS users choose the SD distance according to the criteria (sensitivity or selectivity) more important to them. If the users focus on selectivity, an SD distance of $15 \mathrm{~mm}$ could be a better choice, whereas $25 \mathrm{~mm}$ is suitable for sensitivity as the priority. In addition, if fNIRS users pursue a relatively balanced tradeoff between selectivity and sensitivity, we recommend an SD distance of $20 \mathrm{~mm}$. Our findings also revealed that the optimal SD distance for infants is different from that for adults. Previous adult studies have demonstrated that fNIRS probes should ideally be designed with 30- to 35-mm SD distances, ${ }^{68,73}$ as most light passes through the extra-cerebral superficial tissues at SD distances $<20 \mathrm{~mm} .{ }^{67}$ Compared with that for adults, and the most suitable SD distances for infants are smaller than $30 \mathrm{~mm}$, which indicates that thin superficial layers allow much more photons to pass through the brain tissue.

\subsection{Future Directions and Limitations}

In the current study, we found that the orientation of SD pairs over 10-10 positions had no significant effect on our results, which was consistent with findings from photon propagation in the adult brain. ${ }^{34,38}$ Notably, only the AAL atlas was adopted to obtain the SCC in this study. The orientation of SD pairs may influence the SCC when other infant brain atlases with finer anatomical parcellation are available with advances in MRI techniques.

For the application of this work, the precise optics-based SCC for SD pairs at all 10-10 system scalp locations (Table S3-7 in the Supplementary Material) can be used as a reference table to guide fNIRS users in designing the probe arrangement for targeting specific brain regions, as well as to help them explain fNIRS data obtained according to the 10-10 system. Importantly, the summary of scalp positions whose MLCBR was completely consistent across $0-, 1-$, and 2-yo infants (Table 4) is beneficial for the investigation of functional development for one specific brain region. However, the spatial density of the 10-10 fiducial points for analyzing the optics-based SCC would be relatively sparse because a few brain regions had only a small chance of being corresponded. Our future work will calculate the optics-based SCC by using a denser placement system, ${ }^{74}$ which can guarantee all brain regions with a high probability of being corresponded and estimate underlying brain regions for any arrangement of SD pairs. In addition, the established age-appropriate infant head models and the proposed method for calculating the optics-based SCC can be used to estimate brain regions at any scalp position by virtually placing the SD pairs on the scalp of 0-, 1-, and 2-yo infants, even without infant head structures.

The head tissues within the first postnatal year undergo dramatic growth, such as the total volume of the brain expanding up to double its size. ${ }^{44,75,76}$ Furthermore, several studies have reported the discovery of longitudinal functional brain development during this period. ${ }^{71,77,78}$ However, the 0-yo infant head model used in this study was constructed with a template-based MRI of an approximately 1-month-old infant. Establishing optics-based SCC at a fine temporal scale (every 3 months from birth to 12 months), which is in line with physical development during the first year after birth, it is expected to provide useful information for investigating the longitudinal development of brain functions using the fNIRS technique.

\section{Conclusion}

The present study adopted light propagation analysis to establish a precise optics-based SCC between fNIRS measurement channels (i.e., SD pairs) set on the 10-10 system scalp positions and AAL brain regions in three age-appropriate head models of 0-, 1-, and 2-yo. We found that age-related changes in the SCC metrics, particularly the fiducial points around the longitudinal fissure, correlated with different brain regions across the three age groups. In contrast, the MLCBR was consistent across the three ages for more than half of the fiducial points. Furthermore, we recommend SD distances between 15 and $25 \mathrm{~mm}$ as suitable for fNIRS in infants by simultaneously considering the selectivity and sensitivity of the MLCBR. We sincerely hope that age-appropriate SCC will be a useful reference to guide the design of 
Cai et al.: Correlating functional near-infrared spectroscopy with underlying cortical regions...

fNIRS probes and provide convincing anatomical interpretations of fNIRS data for future infant developmental studies.

\section{Disclosures}

The authors have declared that no conflict of interest exists.

\section{Acknowledgments}

We wish to thank our anonymous reviewers for their assistance in clarifying and enhancing this work.

\section{Code, Data, and Materials Availability}

Data supporting the results reported in the paper can be requested by contacting the corresponding author.

\section{References}

1. J. H. Gilmore et al., "Longitudinal development of cortical and subcortical gray matter from birth to 2 years," Cereb. Cortex 22(11), 2478-2485 (2012).

2. G. Li et al., "Mapping region-specific longitudinal cortical surface expansion from birth to 2 years of age," Cereb. Cortex 23(11), 2724-2733 (2013).

3. G. Li et al., "Mapping longitudinal development of local cortical gyrification in infants from birth to 2 years of age," J. Neurosci. 34(12), 4228-4238 (2014).

4. J. X. Nie et al., "Longitudinal development of cortical thickness, folding, and fiber density networks in the first 2 years of life," Hum. Brain Mapp. 35(8), 3726-3737 (2014).

5. F. Wang et al., "Developmental topography of cortical thickness during infancy," Proc. Natl. Acad. Sci. USA 116(32), 15855-15860 (2019).

6. J. Kagan and N. Herschkowitz, A Young Mind in a Growing Brain, Lawrence Erlbaum Associates, Mahwah, NJ (2005).

7. J. B. Girault et al., "White matter microstructural development and cognitive ability in the first 2 years of life," Hum. Brain Mapp. 40(4), 1195-1210 (2019).

8. S. Lloyd-Fox, A. Blasi, and C. E. Elwell, "Illuminating the developing brain: the past, present and future of functional near infrared spectroscopy," Neurosci. Biobehav. Rev. 34(3), 269-284 (2010).

9. R. E. Vanderwert and C. A. Nelson, "The use of near-infrared spectroscopy in the study of typical and atypical development," Neuroimage 85, 264-271 (2014).

10. R. N. Aslin, M. Shukla, and L. L. Emberson, "Hemodynamic correlates of cognition in human infants," Аnnu. Rev. Psychol. 66, 349-379 (2015).

11. N. M. McDonald and K. L. Perdue, "The infant brain in the social world: moving toward interactive social neuroscience with functional near-infrared spectroscopy," Neurosci. Biobehav. Rev. 87, 38-49 (2018).

12. D. A. Boas et al., "Twenty years of functional near-infrared spectroscopy: introduction for the special issue," Neuroimage 85, 1-5 (2014).

13. F. Scholkmann et al., "A review on continuous wave functional near-infrared spectroscopy and imaging instrumentation and methodology," Neuroimage 85, 6-27 (2014).

14. V. Quaresima and M. Ferrari, "A mini-review on functional near-infrared spectroscopy (fNIRS): Where do we stand, and where should we go?" Photonics-Basel 6(3), 87 (2019).

15. P. Pinti et al., "The present and future use of functional near-infrared spectroscopy (fNIRS) for cognitive neuroscience," Ann. N.Y. Acad. Sci. 1464(1), 5 (2020).

16. V. Jurcak, D. Tsuzuki, and I. Dan, " $10 / 20,10 / 10$, and 10/5 systems revisited: their validity as relative head-surface-based positioning systems," Neuroimage 34(4), 1600-1611 (2007). 
17. Y. Minagawa-Kawai et al., "Neural attunement processes in infants during the acquisition of a language-specific phonemic contrast," J. Neurosci. 27(2), 315-321 (2007).

18. G. Taga and K. Asakawa, "Selectivity and localization of cortical response to auditory and visual stimulation in awake infants aged 2 to 4 months," Neuroimage 36(4), 1246-1252 (2007).

19. S. Lloyd-Fox et al., "Social perception in infancy: a near infrared spectroscopy study," Child Dev. 80(4), 986-999 (2009).

20. M. Okamoto et al., "Three-dimensional probabilistic anatomical cranio-cerebral correlation via the international 10-20 system oriented for transcranial functional brain mapping," Neuroimage 21(1), 99-111 (2004).

21. L. Koessler et al., "Automated cortical projection of EEG sensors: anatomical correlation via the international 10-10 system," Neuroimage 46(1), 64-72 (2009).

22. C. Kabdebon et al., "Anatomical correlations of the international 10-20 sensor placement system in infants," Neuroimage 99, 342-356 (2014).

23. D. Tsuzuki et al., "Macroanatomical landmarks featuring junctions of major sulci and fissures and scalp landmarks based on the international 10-10 system for analyzing lateral cortical development of infants," Front. Neurosci. 11, 394 (2017).

24. S. Lloyd-Fox et al., "Coregistering functional near-infrared spectroscopy with underlying cortical areas in infants," Neurophotonics 1(2), 025006 (2014).

25. L. L. Emberson et al., "Using fNIRS to examine occipital and temporal responses to stimulus repetition in young infants: Evidence of selective frontal cortex involvement," Dev. Cognit. Neurosci. 23, 26-38 (2017).

26. D. Tsuzuki et al., "Virtual spatial registration of stand-alone MRS data to MNI space," Neuroimage 34(4), 1506-1518 (2007).

27. H. Watanabe et al., "Effect of auditory input on activations in infant diverse cortical regions during audiovisual processing," Hum. Brain Mapp. 34(3), 543-565 (2013).

28. Y. Minagawa et al., "Infant word segmentation recruits the cerebral network of phonological short-term memory," Brain Lang. 170, 39-49 (2017).

29. Y. Hakuno et al., "Interactive live fNIRS reveals engagement of the temporoparietal junction in response to social contingency in infants," Neuroimage 218, 116901 (2020).

30. E. Okada et al., "Theoretical and experimental investigation of near-infrared light propagation in a model of the adult head," Appl. Opt. 36(1), 21-31 (1997).

31. M. Firbank, E. Okada, and D. T. Delpy, "A theoretical study of the signal contribution of regions of the adult head to near-infrared spectroscopy studies of visual evoked responses," Neuroimage 8(1), 69-78 (1998).

32. Y. Hoshi et al., "Reevaluation of near-infrared light propagation in the adult human head: implications for functional near-infrared spectroscopy," J. Biomed. Opt. 10(6), 064032 (2005).

33. T. Koyama et al., "Practical and adequate approach to modeling light propagation in an adult head with low-scattering regions by use of diffusion theory," Appl. Opt. 44(11), 2094-2103 (2005).

34. G. E. Strangman, Q. Zhang, and Z. Li, "Scalp and skull influence on near infrared photon propagation in the Colin27 brain template," Neuroimage 85, 136-149 (2014).

35. Y. Fukui, Y. Ajichi, and E. Okada, "Monte Carlo prediction of near-infrared light propagation in realistic adult and neonatal head models," Appl. Opt. 42(16), 2881-2887 (2003).

36. M. Dehaes et al., "Assessment of the frequency-domain multi-distance method to evaluate the brain optical properties: Monte Carlo simulations from neonate to adult," Biomed. Opt. Express 2(3), 552-567 (2011).

37. G. A. Z. Morais, J. B. Balardin, and J. R. Sato, "fNIRS optodes' location decider (fOLD): a toolbox for probe arrangement guided by brain regions-of-interest," Sci Rep 8, 3341 (2018).

38. L. Cai et al., "Targeting brain regions of interest for functional near-infrared spectroscopyscalp-cortex correlation using subject-specific light propagation models," Hum. Brain Mapp. 42, 1969-1986 (2021).

39. G. Taga, F. Homae, and H. Watanabe, "Effects of source-detector distance of near infrared spectroscopy on the measurement of the cortical hemodynamic response in infants," Neuroimage 38(3), 452-460 (2007). 
Cai et al.: Correlating functional near-infrared spectroscopy with underlying cortical regions...

40. F. Shi et al., "Infant brain atlases from neonates to 1-and 2-year-olds," PLoS One 6(4), e18746 (2011).

41. N. Tzourio-Mazoyer et al., "Automated anatomical labeling of activations in SPM using a macroscopic anatomical parcellation of the MNI MRI single-subject brain," Neuroimage 15(1), 273-289 (2002).

42. UNC IDEA Lab, "UNC 0-1-2 Infant Atlases," https://www.nitrc.org/projects/pediatricatlas/ (2020).

43. R. W. Young, "Age changes in the thickness of the scalp in white males," Hum. Biol. 31(1), 74-79 (1959).

44. Z. G. Li et al., "A statistical skull geometry model for children 0-3 years old," PLoS One 10(5), e0127322 (2015).

45. Q. Q. Fang and D. A. Boas, "Tetrahedral mesh generation from volumetric binary and grayscale images," in IEEE Int. Symp. Biomed. Imaging: From Nano to Macro, Vols. 1 and 2, pp. 1142-1145 (2009).

46. S. Brigadoi et al., "A 4D neonatal head model for diffuse optical imaging of pre-term to term infants," Neuroimage 100, 385-394 (2014).

47. A. Liu and B. Joe, "Relationship between tetrahedron shape measures," BIT Numer. Math. 34(2), 268-287 (1994).

48. Optmed Lab, Dartmouth Colledge, "NIRFAST open source software for multi-modal optical molecular imaging," http://www.dartmouth.edu/ nir/nirfast/ (2018).

49. H. Dehghani et al., "Near infrared optical tomography using NIRFAST: algorithm for numerical model and image reconstruction," Commun. Numer. Methods Eng. 25(6), 711-732 (2009).

50. M. Jermyn et al., "Fast segmentation and high-quality three-dimensional volume mesh creation from medical images for diffuse optical tomography," J. Biomed. Opt. 18(8), 086007 (2013).

51. M. Dehaes et al., "Quantitative effect of the neonatal fontanel on synthetic near infrared spectroscopy measurements," Hum. Brain Mapp. 34(4), 878-889 (2013).

52. M. R. Nuwer, "10-10 electrode system for EEG recording," Clin. Neurophysiol. 129(5), 1103 (2018).

53. Y. Oki, H. Kawaguchi, and E. Okada, "Validation of practical diffusion approximation for virtual near infrared spectroscopy using a digital head phantom," Opt. Rev. 16(2), 153-159 (2009).

54. S. R. Arridge, "Photon-measurement density functions. Part I: analytical forms," Appl. Opt. 34(31), 7395-7409 (1995).

55. S. R. Arridge and M. Schweiger, "Photon-measurement density functions. Part 2: finiteelement-method calculations," Appl. Opt. 34(34), 8026-8037 (1995).

56. K. Noguchi et al., "nparLD: an R software package for the nonparametric analysis of longitudinal data in factorial experiments," J. Stat. Software 50(12), 1-23 (2012).

57. S. Olejnik and J. Algina, "Generalized eta and omega squared statistics: measures of effect size for some common research designs," Psychol. Methods 8(4), 434-447 (2003).

58. R. Bakeman, "Recommended effect size statistics for repeated measures designs," Behav. Res. Methods 37(3), 379-384 (2005).

59. S. L. Ferradal et al., "Functional imaging of the developing brain at the bedside using diffuse optical tomography," Cereb. Cortex 26(4), 1558-1568 (2016).

60. A. Custo et al., "Anatomical atlas-guided diffuse optical tomography of brain activation," Neuroimage 49(1), 561-567 (2010).

61. R. J. Cooper et al., "Validating atlas-guided DOT: a comparison of diffuse optical tomography informed by atlas and subject-specific anatomies," Neuroimage 62(3), 1999-2006 (2012).

62. S. L. Ferradal et al., "Atlas-based head modeling and spatial normalization for high-density diffuse optical tomography: in vivo validation against fMRI," Neuroimage 85, 117-126 (2014).

63. S. Gallo, C. A. Vanstone, and H. A. Weiler, "Normative data for bone mass in healthy term infants from birth to 1 year of age," J Osteoporos 2012, 1-8 (2012).

64. H. Delye et al., "Creating a normative database of age-specific 3D geometrical data, bone density, and bone thickness of the developing skull: a pilot study," J. Neurosurg. Pediatr. 16(6), 687-702 (2015). 
65. R. B. Dietrich et al., "MR evaluation of early myelination patterns in normal and developmentally delayed infants," AJR Am. J. Roentgenol. 150(4), 889-896 (1988).

66. L. Spinelli et al., "In vivo measure of neonate brain optical properties and hemodynamic parameters by time-domain near-infrared spectroscopy," Neurophotonics 4(4), 041414 (2017).

67. L. Wang, H. Ayaz, and M. Izzetoglu, "Investigation of the source-detector separation in near infrared spectroscopy for healthy and clinical applications," J. Biophotonics 12(11), e201900175 (2019).

68. G. E. Strangman, Z. Li, and Q. Zhang, "Depth sensitivity and source-detector separations for near infrared spectroscopy based on the Colin27 brain template," PLoS One 8(8), e66319 (2013).

69. L. D. Reyes et al., "The functional brain networks that underlie visual working memory in the first two years of life," Neuroimage 219, 116971 (2020).

70. S. Lloyd-Fox et al., "Cortical specialisation to social stimuli from the first days to the second year of life: a rural Gambian cohort," Dev. Cognit. Neurosci. 25, 92-104 (2017).

71. H. O. Miguel et al., "Infant brain response to affective and discriminative touch: a longitudinal study using fNIRS," Social Neurosci. 14(5), 571-582 (2019).

72. C. Bulgarelli et al., "The developmental trajectory of fronto-temporoparietal connectivity as a proxy of the default mode network: a longitudinal fNIRS investigation," Hum. Brain Mapp. 41(10), 2717-2740 (2020).

73. T. Li, H. Gong, and Q. Luo, "Visualization of light propagation in visible Chinese human head for functional near-infrared spectroscopy," J. Biomed. Opt. 16(4), 045001 (2011).

74. R. Oostenveld and P. Praamstra, "The five percent electrode system for high-resolution EEG and ERP measurements," Clin. Neurophysiol. 112(4), 713-719 (2001).

75. G. Li et al., "Computational neuroanatomy of baby brains: a review," Neuroimage 185, 906-925 (2019).

76. W. Gao et al., "Functional connectivity of the infant human brain: plastic and modifiable," Neuroscientist 23(2), 169-184 (2017).

77. W. Gao et al., "Functional network development during the first year: relative sequence and socioeconomic correlations," Cereb. Cortex 25(9), 2919-2928 (2015).

78. X. Y. Wen et al., "First-year development of modules and hubs in infant brain functional networks," Neuroimage 185, 222-235 (2019).

Lin Cai is a $\mathrm{PhD}$ candidate in the Department of Electronics and Electrical Engineering, Keio University. His research interests mainly focus on the use of fNIRS to investigate cognitive development and the resting-state functional brain network during infancy and childhood. When working on his $\mathrm{PhD}$, he has been investigating the brain mechanisms in early language acquisition at Keio Baby Lab and the modeling of light propagation in infants' heads at Okada Lab.

Eiji Okada is a professor in the Department of Electronics and Electrical Engineering at Keio University. He received his $\mathrm{PhD}$ in electrical engineering from Keio University in 1990. His research interests have involved modeling of light propagation in biological tissue and its application to functional near infrared spectroscopy and diffuse optical imaging.

Yasuyo Minagawa is a professor in the Department of Psychology at Keio University. She received her $\mathrm{PhD}$ in medicine from the University of Tokyo in 2000. Her research examines the development of perception and cognition with a focus on language acquisition and social cognition in infants. To reveal the neural substrates underlying such development, she has successfully applied fNIRS to typically and atypically developing infants. This application extends to real-world neuroimaging such as mother-infant hyperscanning.

Hiroshi Kawaguchi is a senior research scientist at the Human Informatics and Interaction Research Institute, National Institute of Advanced Industrial Science and Technology, Japan. He received his $\mathrm{PhD}$ in engineering from Keio University, Japan. His current research interests include the development of human neuroimaging techniques, such as near-infrared spectroscopy. 